\title{
e-Phaïstos
}

e-Phaïstos Revue d'histoire des techniques / Journal of the history of technology

IX-2 | 2021

Quel objet pour quel musée?

\section{L’informatique au musée national des Techniques de Prague}

Méthodologie de l'exposition « La piste tchèque dans l'histoire de l'informatique » (novembre2020-mai 2021)

Computer Science at the National Museum of Technology in Prague:

Methodology of the exhibition "The Czech Trail in the History of Informatics"

(November 2020-May 2021)

\section{Marcela Efmertová}

\section{(2) OpenEdition}

Journals

\section{Édition électronique}

URL : https://journals.openedition.org/ephaistos/9640

DOI : 10.4000/ephaistos.9640

ISSN : 2552-0741

\section{Éditeur}

IHMC - Institut d'histoire moderne et contemporaine (UMR 8066)

\section{Référence électronique}

Marcela Efmertová, «L'informatique au musée national des Techniques de Prague », e-Phaïstos [En ligne], IX-2 | 2021, mis en ligne le 26 octobre 2021, consulté le 28 octobre 2021. URL : http:// journals.openedition.org/ephaistos/9640; DOI : https://doi.org/10.4000/ephaistos.9640

Ce document a été généré automatiquement le 28 octobre 2021.

Tous droits réservés 


\section{L'informatique au musée national des Techniques de Prague}

Méthodologie de l'exposition « La piste tchèque dans l'histoire de l'informatique » (novembre2020-mai 2021)

Computer Science at the National Museum of Technology in Prague: Methodology of the exhibition "The Czech Trail in the History of Informatics"

(November 2020-May 2021)

\section{Marcela Efmertová}

«Une machine mathématique ne doit pas être une surprise sensationnelle pour un jeune. Il doit se familiariser avec elle en tant qu'équipement habituel, qui est un des moyens de subsistance normaux ", Antonín Svoboda, Première conférence nationale sur les technologies informatiques et organisationnelles, Prague, 1958

« Il n'y a aucune raison pour que quelqu'un souhaite avoir un ordinateur à la maison », Ken Olson, président et fondateur de DEC, 1977

« 640 Ko devraient suffire à tous », Bill Gates, 1981 (disquette 1,44 Mo, disque USB 32 Go, disque

dur 1 To)

1 Les musées et la préservation du patrimoine culturel ont toujours été associés à la formation de la société civile moderne dans le monde tchèque, tant pour la recherche et l'éducation que le renforcement de l'identité culturelle nationale (Janko, Štrbáňová 1988 ; Dolejší, Fučík, Havel et Pernes 2004) ${ }^{1}$. C'est en 1775 que František Josef, comte Kinský (1739-1805), cofondateur de la Société savante privée tchèque à la fin des années 1760 (Petráň 1995) $^{2}$, déposa auprès du greffe commun tchèque et autrichien de Vienne ${ }^{3}$, la première proposition de création du Musée tchèque (Kučera, Kaše et Bělina 2006, Prokeš 1926). Ce projet, qui visait à présenter les richesses naturelles du pays et à encourager leur étude, n'a certes pas abouti. Mais il a initié un mouvement qui s'est 
rapidement concrétisé dans les premières décennies du XIX ${ }^{e}$ siècle. Ainsi, le musée d'Opava (Oesterreichisch-schlesisches Provinzialmuseum), précurseur de l'actuel musée de Silésie ${ }^{4}$ a été fondé en 1814. La même année, débutaient les études préliminaires et les travaux pour un futur musée public à Prague ${ }^{5}$. En 1817, le musée morave ${ }^{6}$ était fondé à Brno, suivi en 1818 par le Musée national à Prague ${ }^{7}$. Puis, un siècle plus tard, en 1908, ce fut la fondation, à Prague toujours, du Musée technique national, l'un des cinq plus anciens musées techniques d'Europe ${ }^{8}$. Toutes ces institutions se sont données pour but de collecter, protéger, mener des recherches scientifiques, rendre les collections accessibles au public et les vulgariser. Cette tradition n'a jamais été interrompue. Elle a conduit au développement substantiel des institutions muséales en République tchèque au cours du dernier quart de siècle.

2 Dans ce domaine, l'État -la République tchèque- est représenté par le ministère de la Culture, qui fournit un soutien systématique et efficace aux institutions établies, leur permettant de conserver leur dynamisme et de les maintenir en constante évolution. L'État protège, recherche, met à la disposition du public et assure la promotion des ressources naturelles, culturelles et techniques nationales à travers les collections des musées. Cette obligation découle notamment de la Charte des droits et libertés fondamentaux ${ }^{9}$, à travers ses articles 33 (droit à l'éducation) et 34 (droit d'accès des citoyens à la richesse culturelle).

\section{Aux origines de l'exposition}

3 Le développement scientifique et technique accéléré par la Seconde Guerre mondiale a conduit à la formulation et au développement rapide de la technologie informatique, de la cybernétique, de la communication et des technologies de l'information. Dans l'histoire du développement technique, nous rencontrons rarement un ou plusieurs domaines qui ont connu un développement aussi fulgurant. Au milieu du $\mathrm{XX}^{\mathrm{e}}$ siècle, la technologie informatique regroupait un ensemble d'outils pour assurer notamment des calculs rapides et pratiques. Il s'agit aujourd'hui de technologies de traitement, de stockage et de transmission de différents types d'informations (numériques, textuelles, graphiques, acoustiques...). Partant d'appareils uniques, l'informatique a fini par devenir un outil universel. Le développement complexe et sinueux des technologies de l'information et de l'informatique constitue désormais l'épine dorsale de tous les secteurs de l'activité humaine. Les technologies informatiques sont devenues un élément essentiel de la vie quotidienne. Condition préalable à la performance de nombreuses professions, elles sont également couramment utilisées dans un large éventail d'activités de loisirs. Pour autant, seuls les spécialistes connaissent les principes de fonctionnement de l'ordinateur. Une poignée d'experts, d'historiens et de techniciens, voire de passionnés ou de collectionneurs privés, maîtrise cette histoire.

4 Partie prenante de cette évolution technologique depuis ses débuts, la Tchécoslovaquie a engrangé des résultats significatifs après 1945, principalement grâce à l'équipe d'Antonín Svoboda : Jan G. Oblonský, Vladimír Vand, Jiř̌́ G. Klir, Zdeněk Korvas, Květa Korvasová et leurs successeurs sur plusieurs générations, par exemple Marcel Jiřina, Karel Krištoufek. Ces chercheurs ont œuvré dans le cadre de l'Institut de recherche sur les machines mathématiques (VÚMS, Výzkumný ústav matematických strojů), qui fut d'abord rattaché à l'Académie tchécoslovaque des sciences, puis à la Faculté d'électricité de l'Université polytechnique de Prague. Pour autant, la qualité de ces 
travaux de recherche et la valeur du patrimoine scientifique n'ont pas conduit à la création d'un musée permanent de l'informatique en République tchèque. Les seules initiatives furent locales ou privées ${ }^{10}$. L'exposition « La piste tchèque dans l'histoire de l'informatique ", qui s'est tenue au musée national de Prague, de novembre 2020 à mai 2021 (voir fig.1), est donc la première grande réalisation faite à l'échelle nationale, sur ce thème majeur qu'est l'histoire de la technologie informatique.

Fig.1. Entrée dans l'exposition La piste tchèque dans l'histoire de l'informatique, au musée national technique de Prague

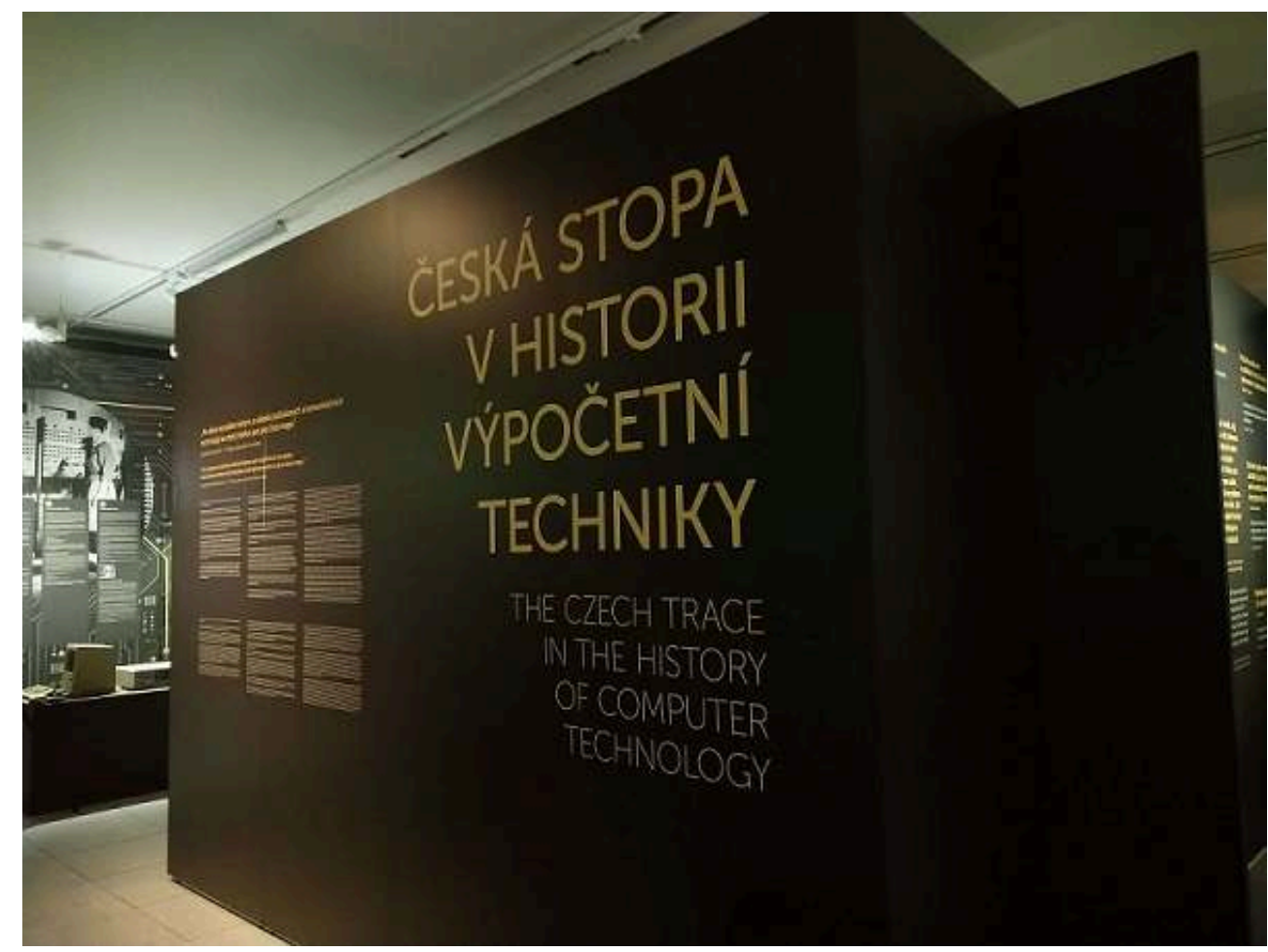

L'exposition, préparée par le Laboratoire d’histoire (électro)technique de génie (électrique) de la Faculté d'électricité de l'Université polytechnique tchèque de Prague en coopération avec le Musée technique national, est consacrée au développement historique de la technologie informatique et est divisée en trois parties. La première section, intitulée De l'abaque au smartphone, traite du développement général de la technologie informatique dans le monde. La deuxième partie, intitulée l'École tchécoslovaque d'informatique, présente le développement de la technologie informatique tchécoslovaque liée principalement à Antonín Svoboda et à l'Institut de recherche sur les machines mathématiques (VúMS, en tchèque). Elle met l'accent sur la contribution tchèque/tchécoslovaque au développement des technologies de l'information. Dans la troisième section, intitulée Microordinateurs, Internet et réalité virtuelle, le visiteur trouve une sélection de micro-ordinateurs domestiques, en particulier ceux des années 1980, avec la possibilité d'essayer des jeux informatiques d'époque et de découvrir l'empreinte tchèque dans le domaine des technologies de l'information.

Cliché Marcela Efmertová

5 L'exposition prend ses racines dans le cadre du projet du ministère de la Culture de la République tchèque NAKI II. DG18P02OVV052 Siècle de l'information: le monde de l'informatique et de l'électrotechnique - le monde de l'informatique en nous, en l'honneur du $70^{\mathrm{e}}$ anniversaire de la création de la Faculté d'électricité de l'Université polytechnique tchèque de Prague. 


\section{Quelle méthodologie?}

6 Il a été décidé d'adopter la méthodologie du comité international spécialisé de l'ICOM (Efmertová et al., 2019). Les travaux préparatoires de conception et de collecte du matériel ont été réalisés selon cette référence méthodologique certifiée (Efmertová et al. 2019), sur la base des objectifs suivants :

- classification professionnelle et recherche scientifique en informatique, cybernétique, communication et technologies de l'information pour les collections de musées en République tchèque ;

- évaluation des conditions climatiques et lumineuses, des influences chimiques dans les institutions muséales pour les expositions informatiques;

- consultation et conseils dans le domaine de la documentation et de la collecte des dispositifs d'information et informatiques, mais aussi de leur stockage dans des dépôts appropriés ;

- suivi continu des sources de documentation (par exemple des manuels) pour les technologies informatiques et de l'information;

- service éducatif et promotion des expositions (conférences, publications);

- coopération autour du fonds collecté avec des institutions spécialisées, des collectionneurs privés et les musées techniques tchèques ou étrangers.

\section{Quel modèle muséal ?}

7 La conception de la scénographie a bénéficié des compétences de l'équipe de la Faculté d'électricité de l'Université polytechnique de Prague, partie prenante du projet muséal depuis ses débuts dans les années 2016-2018 ainsi que de l'expérience muséographique des musées tchèques et slovaques, et de celle des musées étrangers présentant des collections similaires (France, Grande-Bretagne, États-Unis et Allemagne).

Aux États-Unis, plus précisément en Californie, le Computer History Museum de Mountain View $^{11}$ est essentiel pour disposer d'une compréhension globale de l'évolution des technologies informatiques et de l'information, des premières réalisations à nos jours. Intitulée " Revolution: The First 2000 Years of Computing ", l'exposition couvre 25000 pieds carrés $\left(7620 \mathrm{~m}^{2}\right)$. Elle compte 19 galeries, 1100 artefacts ainsi qu'une série d'expériences multimédias originales qui présentent l'histoire de l'informatique, des origines aux smartphones, en passant par divers appareils, logiciels et une multitude de photographies $^{12}$.

En Angleterre, au London Science Museum, des démonstrations de technologies modernes en relation avec l'informatique, sont intégrées à l'exposition «Espace et création du monde moderne $»^{13}$. En Allemagne, le Heinz Nixdorf MuseumsForum (HNF) de Paderborn est un musée thématique axé sur les différentes étapes du développement de l'informatique. Ce musée couvre 5000 ans d'histoire à travers un voyage passionnant qui permet de découvrir des inventeurs, des entrepreneurs, des machines anciennes, mais aussi les dernières évolutions techniques ${ }^{14}$. Pour autant, il se démarque des autres en se concentrant sur la propre production d'une entreprise : Nixdorf.

En France, à partir de 2003, la protection des objets a été étendue au patrimoine scientifique et technique contemporain, ce qui a donné naissance à la mission PATSTEC de protection et de valorisation des objets relatifs aux sciences et technologies du $\mathrm{XX}^{\mathrm{e}}$ siècle. De nouvelles compétences sont apparues dans le domaine de la gestion des musées informatiques. Le musée de l'Informatique de Sophia-Antipolis, près de Nice, en 
constitue un exemple marquant ${ }^{15}$. L'objectif était de créer des collections raisonnées, de contribuer aux débats sur la culture scientifique et technique et de définir les conditions du transfert culturel de la science et de la technologie vers les musées et leurs collections. Autre exemple, le musée installé en 2008-2010, dans la Grande Arche de La Défense, à Paris, grâce à Philippe Nieuwbourg ${ }^{16}$. Ce fut un des premiers musées modernes de l'information, de la cybernétique et de l'informatique. Il avait pour ambition de replacer les objets, leur utilisation et les échecs éventuels, dans leur contexte historique. Suivant ce modèle, des expositions numériques, mais aussi physiques ont vu le jour dans d'autres villes françaises. Ainsi, à Grenoble, le musée informatique ouvert par l'Association pour un Conservatoire de l'Informatique et de la Télématique (ACONIT), s'est donné pour devise: "Connaitre le passé, comprendre le présent, pour imaginer l'avenir». Le musée propose différents types de programmes éducatifs et interactifs associés à 200 équipements électroniques. Il traite notamment de l'origine et de l'histoire d'Internet depuis $1942^{17}$.

11 D'autres lieux ont été étudiés, comme le Deutsches Museum de München et le Landesmuseum Für Technik und Arbeit de Mannheim, en Allemagne; le Technisches Museum à Wien en Autriche; le Museo Nazionale Scienca et le Leonardo da Vinci à Milano en Italie; le Swiss Science Center, Technorama, à Winterthur en Suisse; et, enfin, le Tokyo National Art Center au Japon.

12 En fin de compte, il a été décidé de concevoir l'exposition de Prague en prenant pour principe que les musées ne sont plus de simples dépôts qui stockent et organisent les collections. Ils doivent s'appuyer sur un certain nombre de fonctions sociales, parfois incompatibles les unes avec les autres : les collections doivent être protégées ; le public doit recevoir l'information qu'elles contiennent, et donc, elles doivent être exposées. De ce fait, les musées constituent un lieu de dialogue entre l'expert, le conservateur, le médiateur et le visiteur. Et dans le cas des musées consacrées à l'informatique, ce dialogue est d'autant plus indispensable.

\section{Quelles archives ?}

Au plan mondial, les archives concernant la cybernétique et l'informatique se trouvent généralement dans les institutions muséales susmentionnées. En ce qui concerne l'informatique tchèque ${ }^{18}$, les archives sont conservées au sein des fonds suivants :

- Les Archives du Musée technique national (fonds de l'Institut de Recherche des machines mathématiques, fonds personnels) ;

- Les Archives nationales (fonds du ministère de l'Ingénierie de précision, du ministère de l'Ingénierie générale, ainsi que les résolutions des activités du Parti communiste - fonds du Comité central 1945-1989, Prague-présidium 1962-1966, 1966-1971, etc.) ;

- Les Archives de l'Université polytechnique de Prague (fonds personnels, plus particulièrement celui d'Antonín Svoboda) ;

- Les Archives de l'Université polytechnique de Brno (fonds personnels - collecte de documents biographiques, fonds de l'Institut de génie électrique théorique et expérimental, université des Techniques du Dr. Edvard Beneš) ;

- Les Archives de l'Académie des sciences de la République tchèque (fonds de l'Institut de théorie de l'information et d'automatisation, de gestion et d'administration, du Comité du présidium de l'Académie des sciences tchécoslovaque) et éventuellement les Archives de 
collectionneurs privés, dont les plus célèbres en République tchèque sont celles de M. Petr Váradi ${ }^{19}$ et M. Ing. Michal Suchánek ${ }^{20}$. technologie informatique peuvent être divisée en œuvres ayant leur propre objectif. Outre des ouvrages publiés par des historiens, ils peuvent correspondre à des notes de chercheurs, des journaux de travail, etc., ou des imprimés d'entreprises liées à la production (registres de l'entreprise, modes d'emploi, manuels, catalogues, etc.).

\section{L'exposition : scénographie et parcours muséal}

\section{Le parcours muséal}

Le parcours muséal a distingué trois parties, selon une structure définie et élaborée par Efmertová et al., 201921. Intitulée Du boulier au Smartphone, la première section évoque le développement de la technologie informatique dans le monde ${ }^{22}$. La deuxième partie, intitulée École informatique tchécoslovaque, présente l'histoire de la technologie informatique tchécoslovaque, en particulier par le prisme d'Antonín Svoboda (Efmertová 1998) et celui de VUMS, l'Institut de recherche des machines mathématiques (Klír, Vysoký 2007) (fig.2, 2a, 2b).

Fig.2. Regard sur la salle d'exposition La piste tchèque dans l'histoire de l'informatique

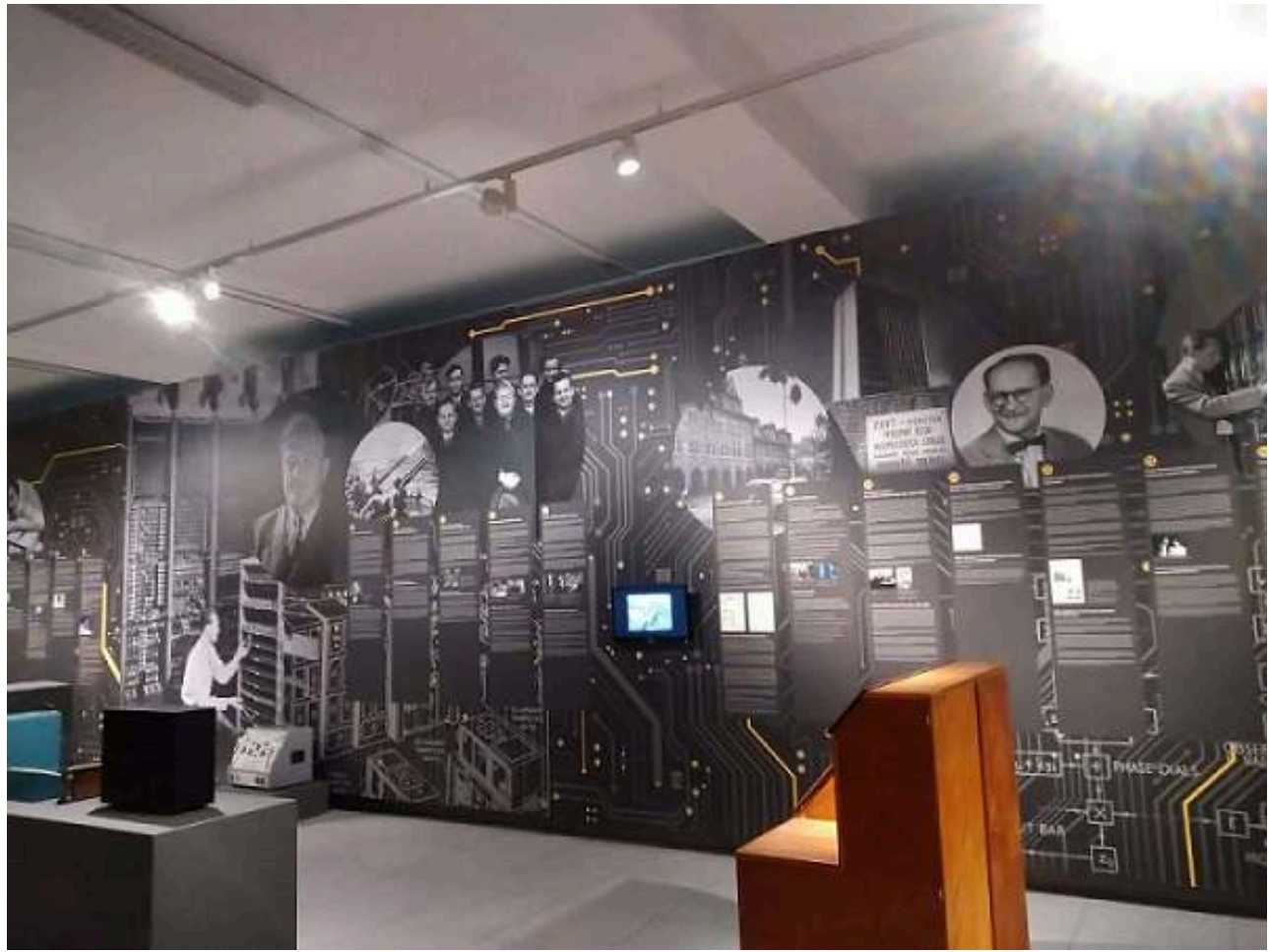

Le mur de l'exposition est dédié au développement de l'Institut de recherche sur les machines mathématiques, où l'ordinateur tchécoslovaque SAPO (sAmočinný Počitač) a été créé après 1945, et aux personnalités associées.

Cliché Marcela Efmertová 


\section{Fig.2a. Antonín Svoboda}

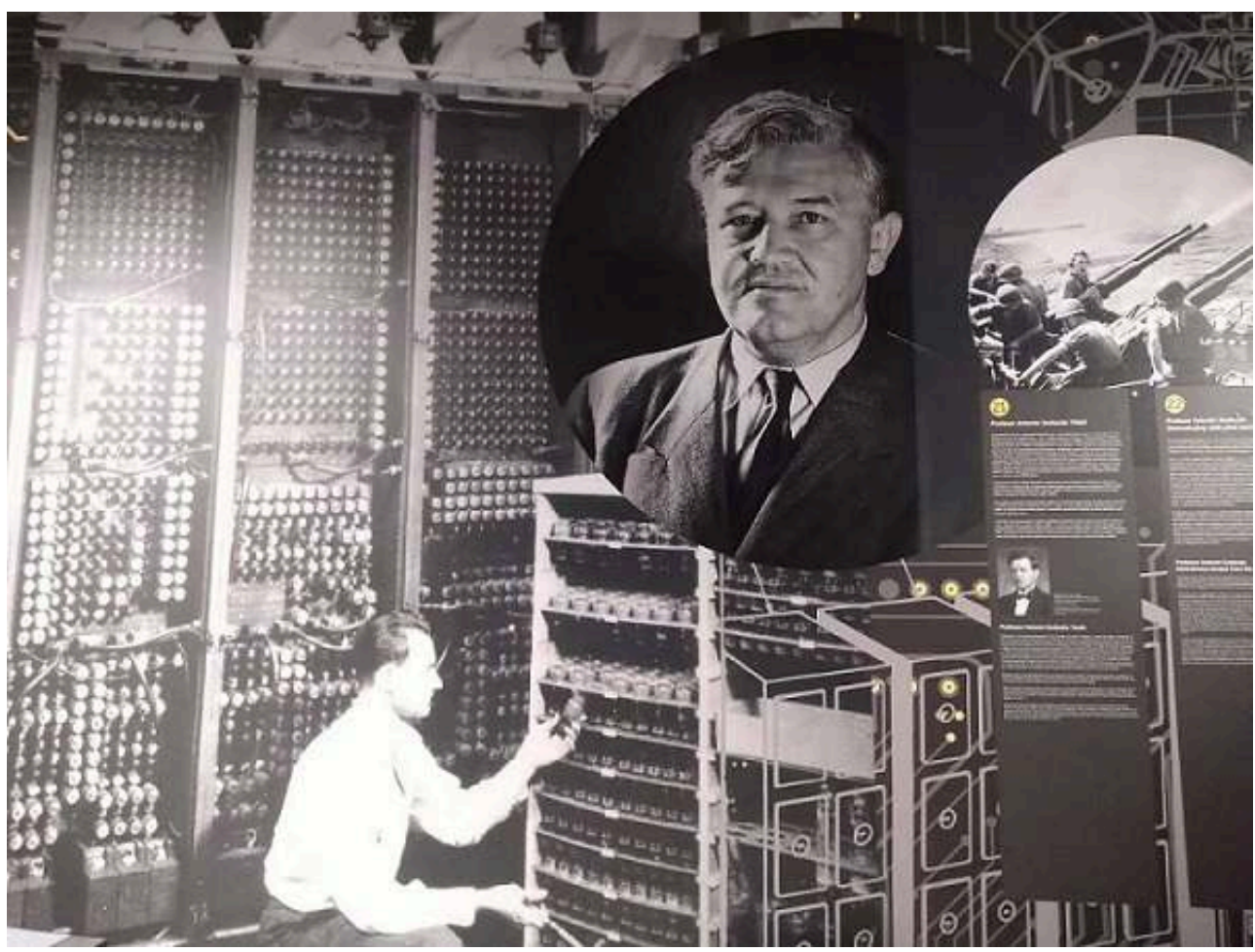

Le principal personnage était Antonín Svoboda (1907-1980, portrait de gauche), un excellent concepteur informatique avec une expérience acquise aux États-Unis pendant la Seconde Guerre mondiale. II fut l'un des pionniers de la société de l'information en Tchécoslovaquie. Le régime communiste a essayé de l'effacer de la mémoire des citoyens tchécoslovaques après son émigration en 1964. II a connu d'autres succès à l'UCLA, aux États-Unis.

Cliché Marcela Efmertová 
Fig.2 b. Antonín Svoboda et son collaborateur Jan Oblonský, au travail sur le SAPO

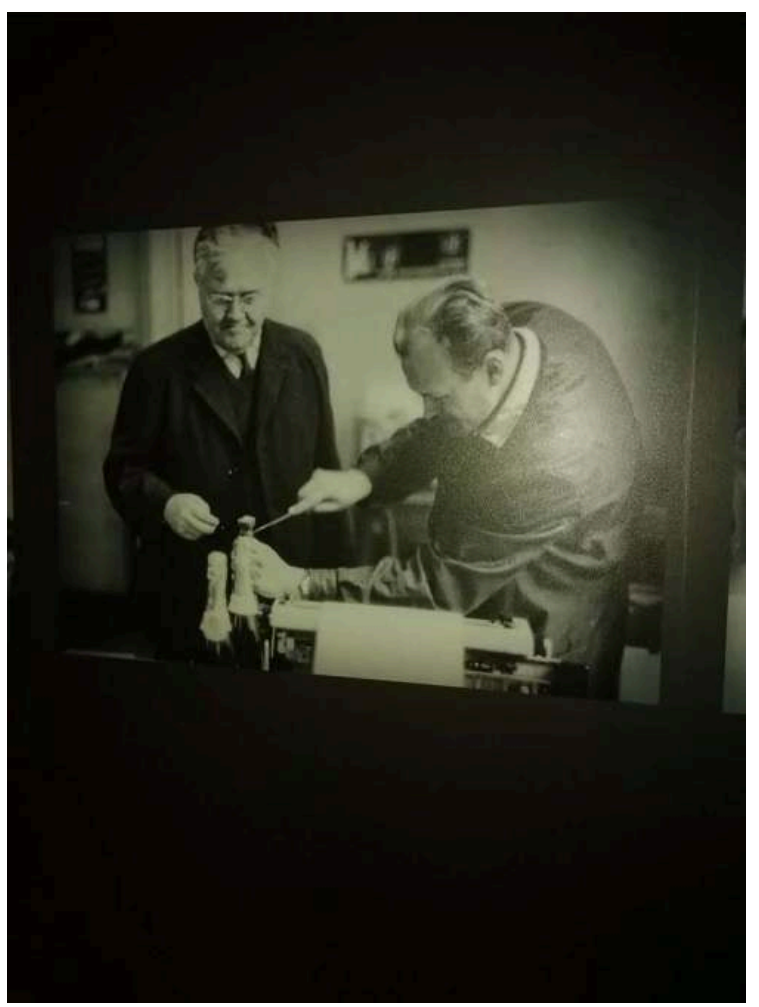

Vladimír Vand (1911-1968) a émigré en France avec J. Oblonský, le 1er mai 1939. Ils travaillèrent ensemble sur des viseurs de défense anti-aérienne pour l'armée française. Durant la Débâcle, en 1940, Svoboda s'est enfuit aux États-Unis et Vand en Grande-Bretagne. Le mur présente également d'autres collaborateurs de A. Svoboda et la place de I'Institut de recherche sur les machines mathématiques dans l'approche scientifique en Tchécoslovaquie, notamment dans les années 1950 et 1960.

Cliché Marcela Efmertová

Cette section met l'accent sur les contributions tchèques / tchécoslovaques. Les étapes de la diffusion de la technologie informatique en Tchécoslovaquie ont été définies comme suit : la période initiale (innovante) de 1947-1958; la phase de recherche et de production entre 1958 et 1968 (fig.4, 5, 6 et 7); puis au sein du Conseil d'assistance économique mutuelle (CAEM, encore appelé COMECON), entre 1968 et 1989 ; enfin la recherche et le développement après la révolution de velours de novembre 1989.

La troisième section, intitulée Micro-ordinateurs et Internet (PAVT 2014), est consacrée aux micro-ordinateurs locaux des années 1990 (fig.3). Elle offre la possibilité d'essayer des jeux informatiques et des technologies informatiques actuelles. La conclusion de l'exposition mène à une réflexion plus approfondie sur l'incroyable saut technologique accompli au cours des soixante dernières années. 
Fig.3. Les micro-ordinateurs tchécoslovaques

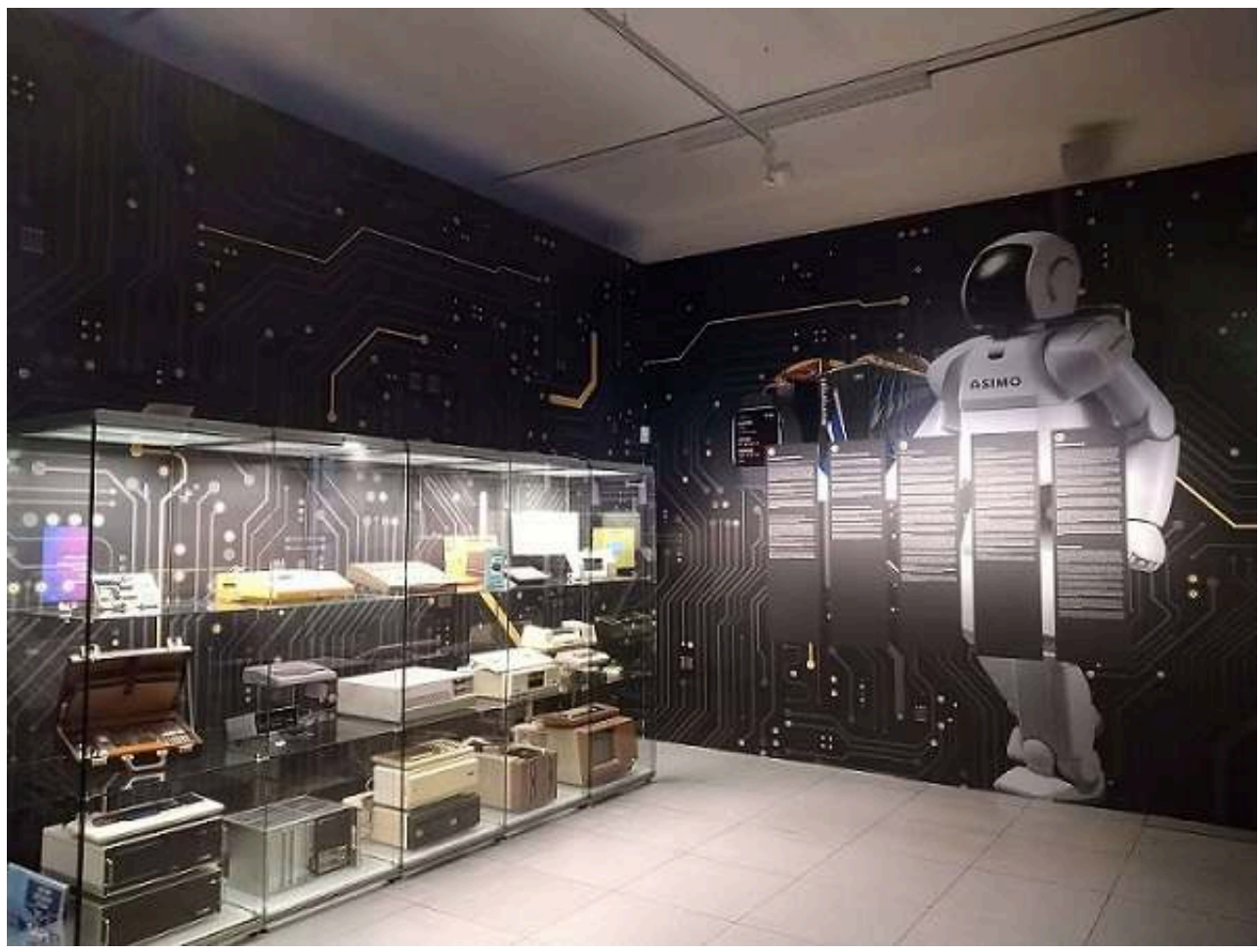

La photographie présente des micro-ordinateurs tchécoslovaques utilisés dans la vie quotidienne (à la maison, dans les bureaux) et dans tous les types d'écoles en Tchécoslovaquie dans les années 1980

(Didaktik, Mato, PMD, etc.). Elle met également en avant des robots contemporains et des informations sur la réalité virtuelle et l'intelligence artificielle.

Cliché Marcela Efmertová 
Fig.3a. Le TESLA PMD-85, ordinateur personnel 8 bits produit à partir de 1985 par les entreprises TESLA Pieštany et TESLA Bratislava

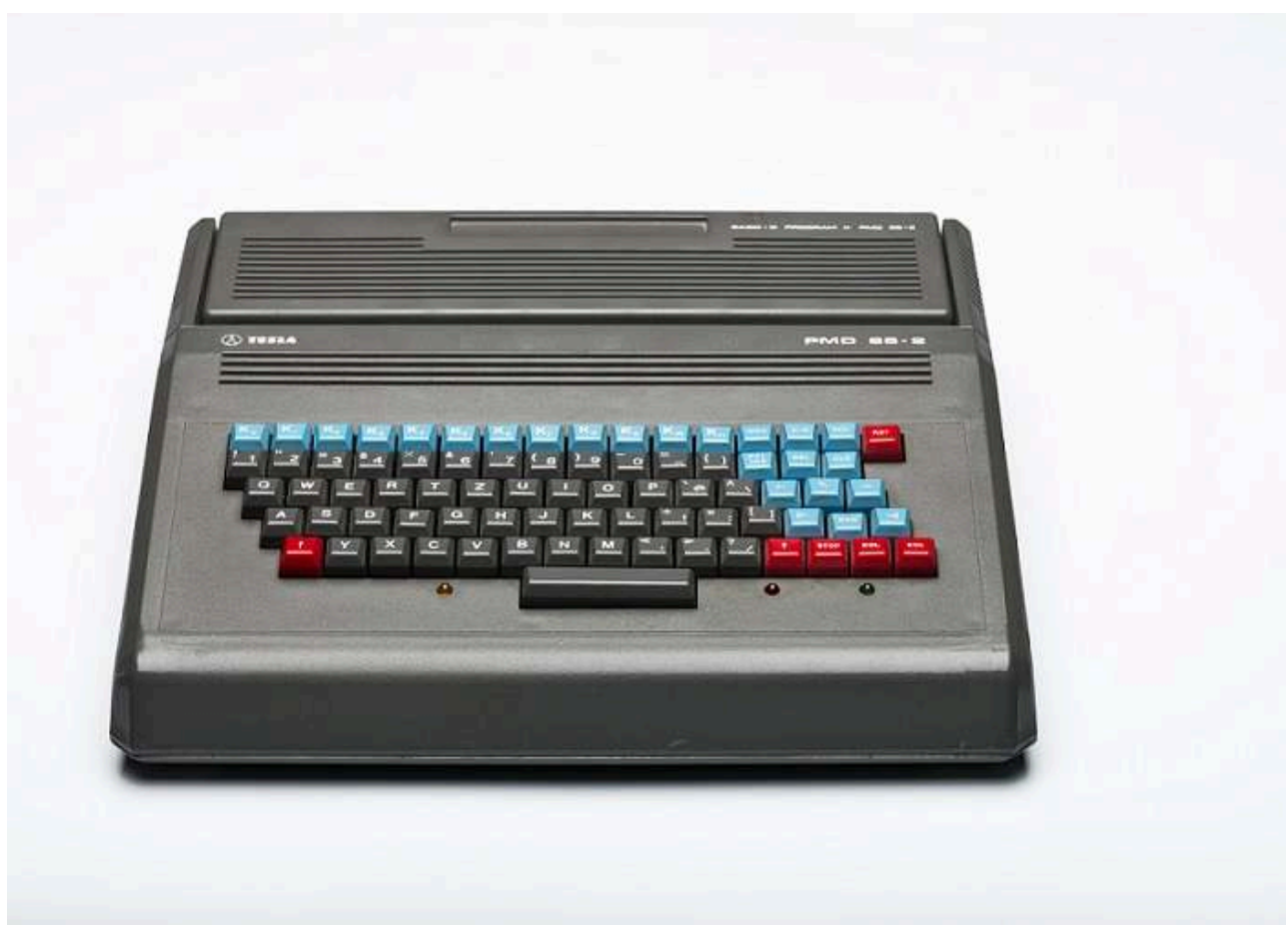

Produit sous la direction de l'ingénieur Roman Kišš, cet ordinateur était destiné principalement aux applications scolaires. Les premiers jeux vidéo tchécoslovaques ont été conçus pour le PMD 85. Son ossature était la version tchécoslovaque du microprocesseur 8088, désigné sous le vocable MHB80A. Sa mémoire de base était de $48 \mathrm{kB}$ de RAM, sa mémoire ROM de 4 ou 8kB (en option). Équipée d'un clavier intégré à 78 touches, la sortie du micro-ordinateur pouvait être reliée à un téléviseur ordinaire en noir et blanc ou à un moniteur couleur (signaux $R, G, B$, régime graphique avec un motif de points de $288 \times 256$ points). Un magnétophone à cassette ordinaire servait de rangement externe. Contenant une interface pour le bus IMS 2 (HP-IB), la machine avait également une sortie pour une imprimante. L'ordinateur est doté d'un module matériel comprenant le langage de programmation Basic G.

Cliché Marcela Efmertová 


\section{Fig.3b - Ordinateur domestique Didaktik Gama}

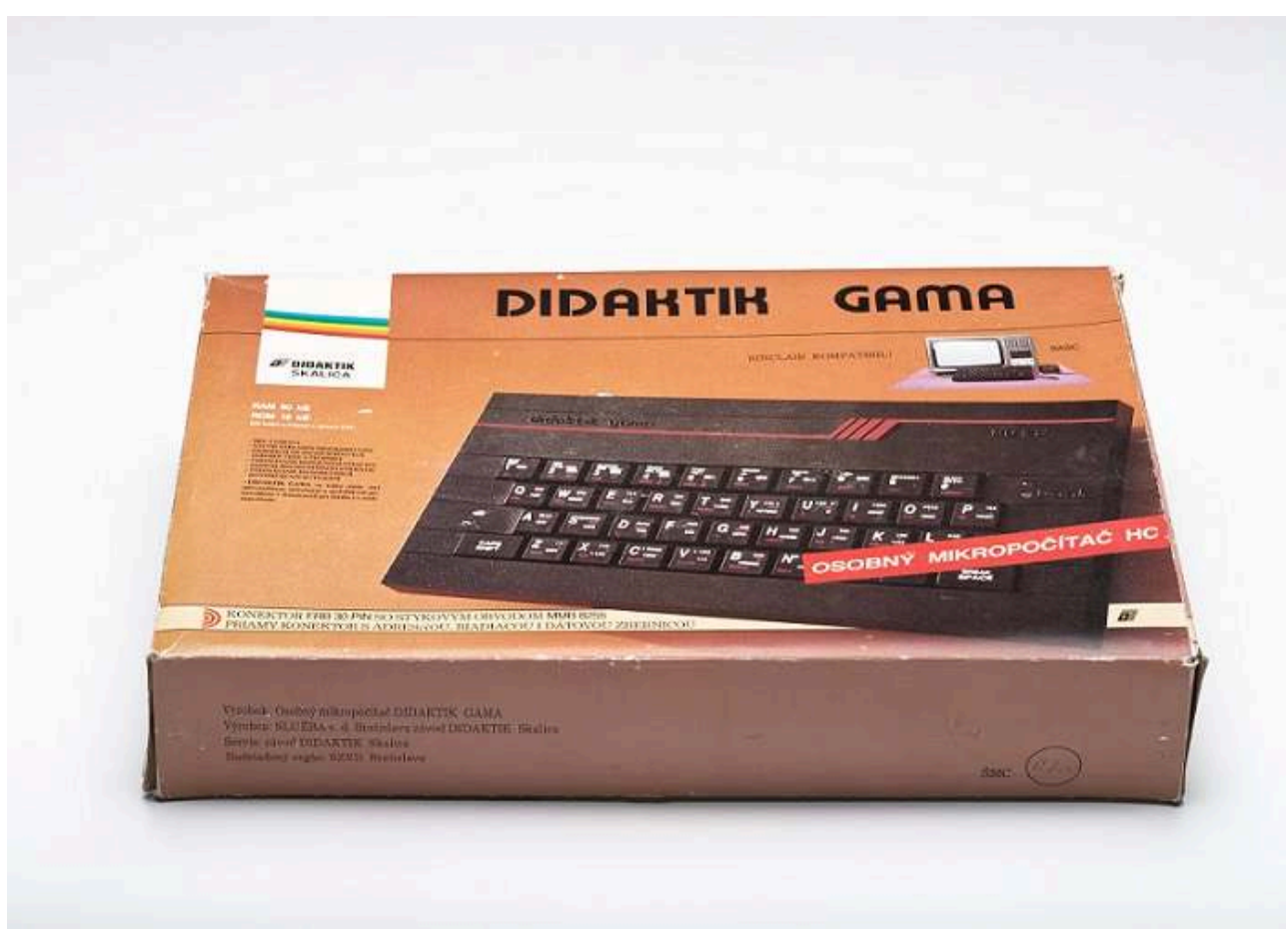

Il s'agit de l'un des ordinateurs personnels 8 bits de la maison produits par la coopérative slovaque Didaktik Skalica. Dans sa version originale, c'était un ordinateur PMD 85 amélioré. Ce micro-ordinateur 8 bits est dérivé du populaire ordinateur ZX Spectrum $48 \mathrm{~K}$. Le micro-ordinateur employait le microprocesseur U880D, un équivalent du Zilog Z80 de production est-allemande. Contrairement à la mémoire RAM de $48 \mathrm{kB}$ du Spectrum, Didaktik affiche une mémoire complète de $80 \mathrm{kB}$, avec une mémoire ROM de 16 kB. Le clavier était livré avec des touches classiques. Par rapport à ZX Spectrum, doté d'une sortie pour un seul bus système, Didaktik offrait l'avantage d'une interface parallèle classique (avec le circuit 8255), facilitant la connexion d'un certain nombre de périphériques. La section d'affichage ne faisait pas partie de l'ordinateur ; on utilisait plutôt un téléviseur ordinaire (entrée soit UHF, soit vidéo directe, affichage 256 points, 8 couleurs avec le système PAL).

Cliché Marcela Efmertová 


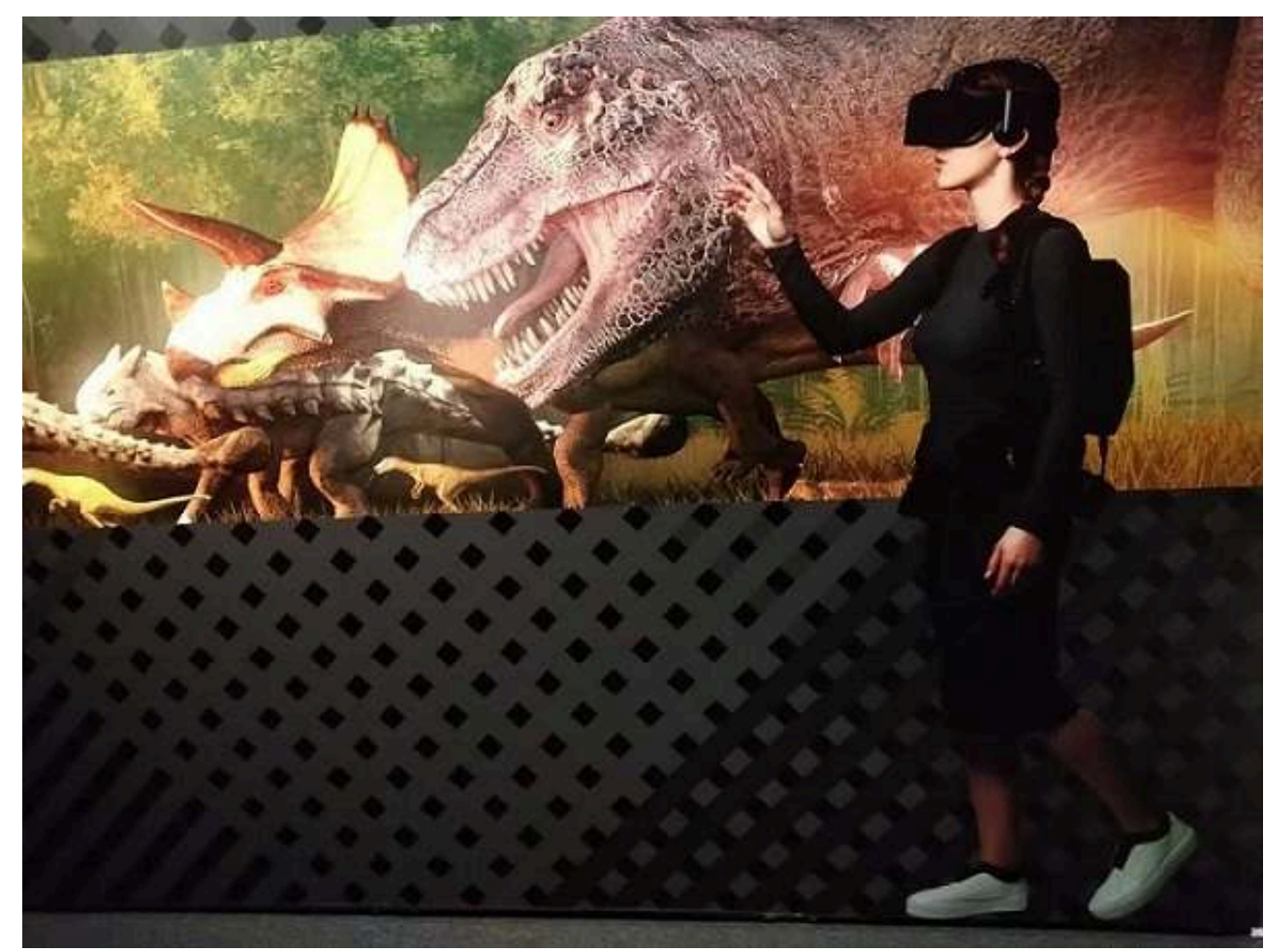

La République tchèque est réputée pour ses jeux virtuels et vidéo. En 1999, est créé le jeu de guerre d'action tactique Hidden and Dangerous. Par la suite, sont sortis un certain nombre de jeux, tels Vietcong et la série de jeux à succès Mafia (2002), Mafia II (DLC, 2010), Mafia III (2016). Cette aventure d'action mafieuse primée est toujours populaire avec une réédition de 2020 : Definitive Edition. Une coopération approfondie avec l'éditeur Take-Two Interactive a débouché sur la sortie entre autres d'un RPG en monde ouvert appelé Kingdom Come: Deliverance, qui se déroule en 1403 en République tchèque. C'est une aventure historique réussie et pleine d'action. Tous les jeux ont une stylisation artistique très forte, un système sophistiqué d'énigmes, une musique et des sons distinctifs. Grâce aux commandes intelligentes et intuitives, les jeux sont populaires non seulement auprès des enfants, mais aussi des adultes, même non-joueurs. Ces jeux peuvent être utilisés non seulement sur des PC, mais également sur des appareils mobiles et des consoles de jeux PlayStation, Xbox et Nintendo Switch.

Cliché Marcela Efmertová

\section{Le sens donné à la scénographie}

18 Il a été décidé de faire comprendre par la scénographie à quel point les équipements informatiques ont changé les événements politiques, sociaux et culturels. Mais comprendre l'impact des technologies informatiques sur la société suppose de caractériser les changements (nouvelles idées techniques, innovations et améliorations) et les développements d'équipements (machines, appareils et leurs périphériques, pièces et composants) qui ont apporté de nouvelles connaissances et compétences aux professionnels et au grand public.

19 Dans la plupart des équipements professionnels, le processus de développement de la technologie informatique est divisé en générations informatiques (Ifrah 2001) selon sept étapes (génération 0 , génération 1 , génération 2, génération 3, génération 3,5, génération 4 et génération 5). Pour les machines de première génération, les grands ordinateurs centraux composés de tubes à vide, l'exposition présente les réalisations 
tchèques, dont l'ordinateur autonome - samočinný počítač - SAPO (fig.4) et l'ordinateur EPOS - elektronický počítacístroj - I et II (fig.5).

Fig. 4. L'ordinateur autonome SAPO (Samočinný počítač)

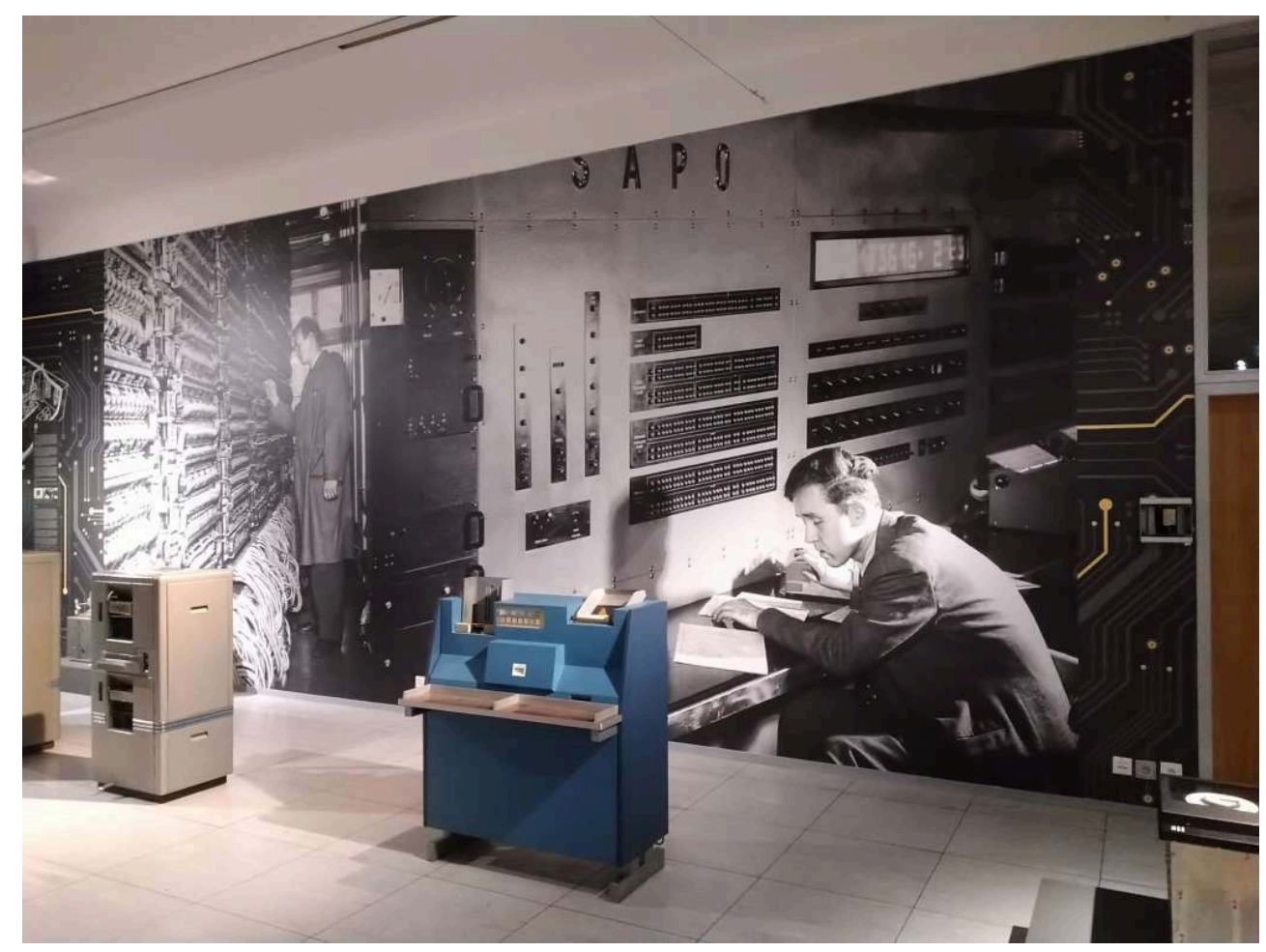




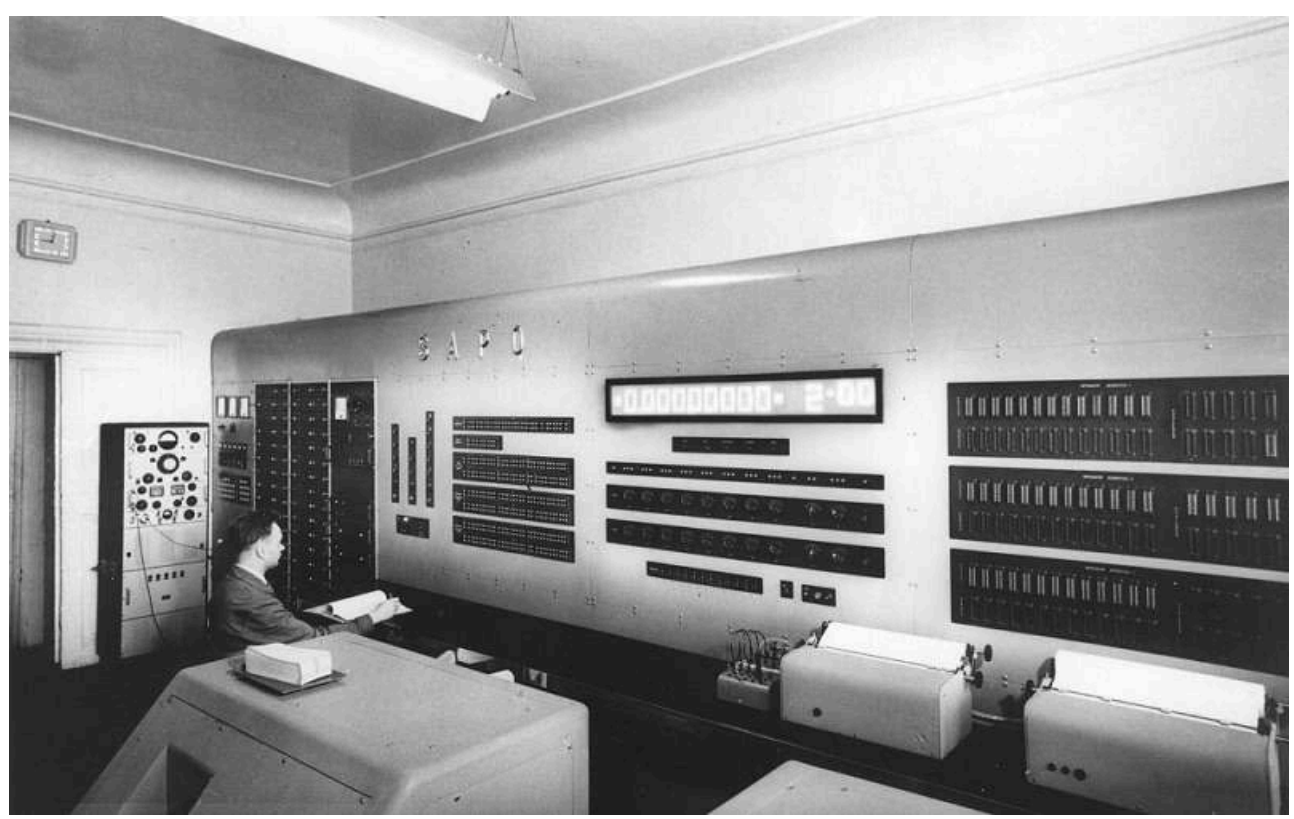

SAPO a été le premier ordinateur fabriqué en Tchécoslovaquie. Il a été utilisé de 1958 à 1960. C'était un prototype expérimental. L'ordinateur était basé sur un relais. Au moment de la mise en service, il occupait une superficie de 100m2, au 3 Loretánské náměstí à Prague. Sa conception a commencé en 1950 à l'Institut de recherche sur les machines mathématiques. Le chef de projet était Antonín Svoboda, qui avait l'expérience des premiers ordinateurs américains qu'il avait connus pendant la Seconde Guerre mondiale au moment de son séjour au MIT. Le projet a été achevé en 1951.

Cependant, la mise en œuvre effective de l'ordinateur relais n'a réussi qu'après plusieurs années. II a été mis en service au tournant des années 1957-1958. II a été utilisé jusqu'en février 1960, époque d'un incendie dans la partie relais de la machine. L'ordinateur n'a pas été restauré, car les recherches se sont concentrées sur la machine à calculer électronique (EPOS).

Cliché Marcela Efmertová 
Fig. 5. L'ordinateur EPOS 1 (Elektronický POčítací Stroj)

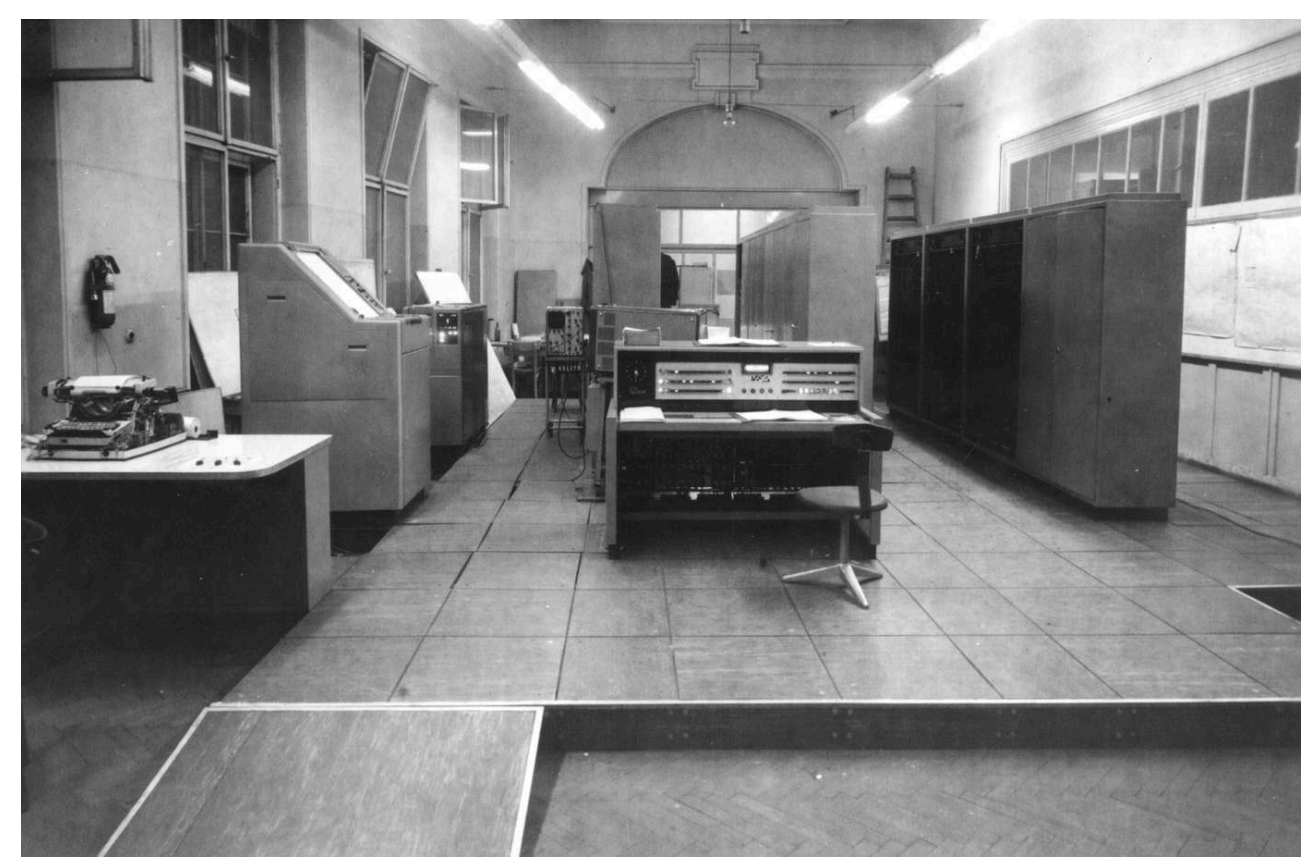

La machine de support informatique électronique - EPOS 1 - a été construite sous la direction de Jan Oblonský et Antonín Svoboda, en coopération avec V. Chlouba, M. Valach, Z. Korvas, V. Bubeník, J. Sedlak, E. Kinder, J. Jůza et J. Imlauf. Contrairement aux ordinateurs à relais de génération zéro, il était déjà équipé de tubes à vide et appartenait à la catégorie des performances moyennes. II a été multi-programmé et conçu spécialement pour les calculs scientifiques et techniques. Par conséquent, il avait également des instructions pour travailler avec des nombres à virgule flottante. Le prototype a été relancé en 1963. L'assemblage informatique se composait d'une unité de base avec une table de contrôle, une unité arithmétique et une mémoire rapide en ferrite. La mémoire supplémentaire était une mémoire à tambour magnétique et à bande magnétique. Les cartes perforées étaient de quatrevingt-dix colonnes. Le modèle fonctionnel, qui a ensuite été introduit dans l'industrie, comprenait 8000 tubes à vide, contre 2000 pour le modèle d'origine, et une consommation électrique de $14 \mathrm{~kW}$. Au total, trois prototypes ont été produits à l'IRMM à partir de 1963 ; l'un d'entre eux a fonctionné de manière fiable à l'état-major de l'armée tchécoslovaque jusqu'en 1974.

Laboratoire d'histoire (électro)technique de la Faculté d'électricité de I'Université polytechnique de Prague

Pour le fonctionnement de ces machines, il a fallu développer des langages de programmation (FORTRAN, COBOL, ALGOL) liés à la seconde génération d'ordinateurs (fig.6). 


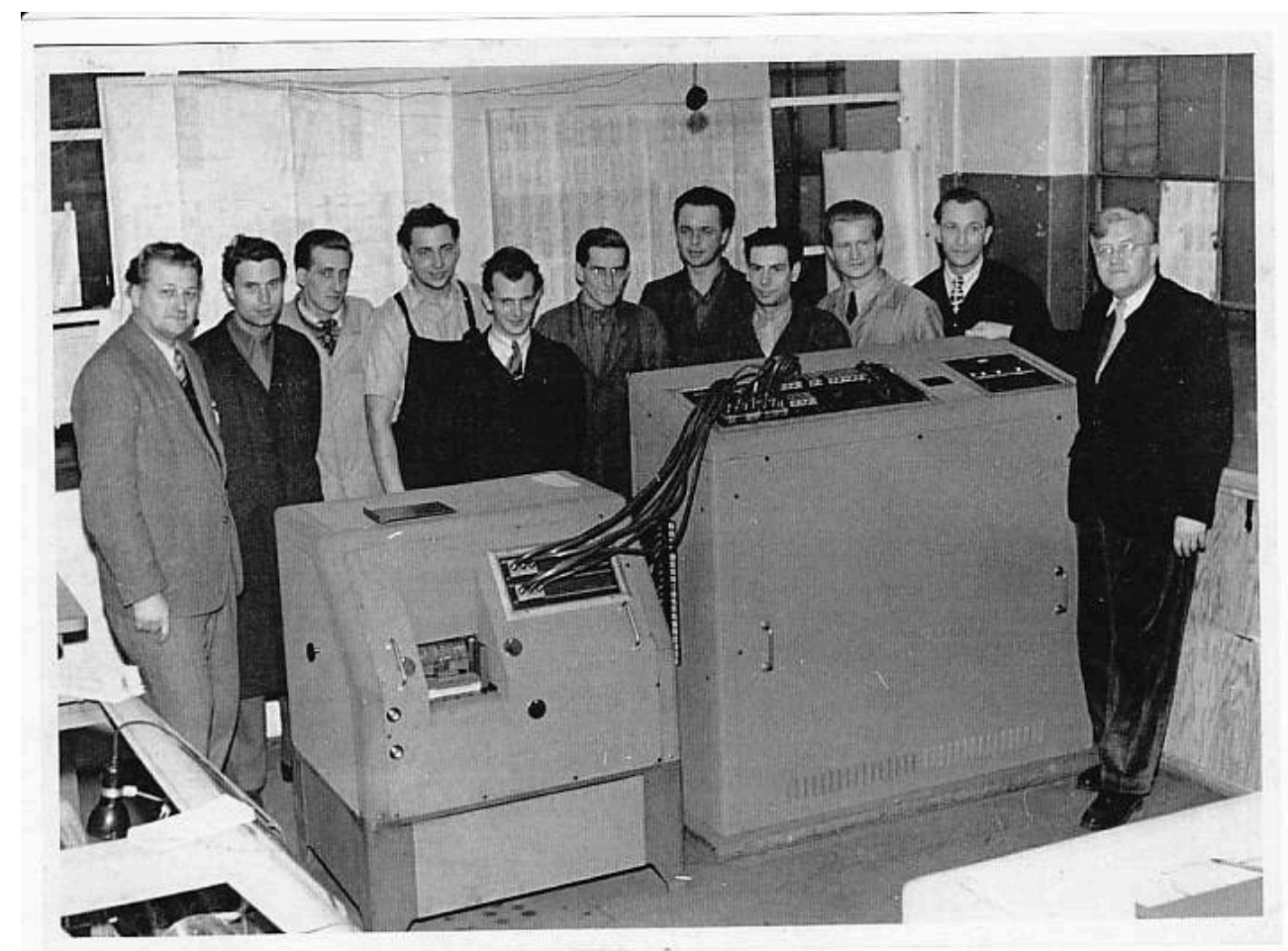

Le calculateur à cartes perforées de la société Aritma, de type 521, est composé de deux unités indépendantes reliées entre elles par un câblage. L'une contient un dispositif de lecture et de poinçonnage pour les cartes perforées, l'autre comprend une unité arithmétique distincte avec logique de relais. II s'agissait d'une calculatrice relais semi-automatique traitant automatiquement les données sur les cartes perforées selon une opération préalablement sélectionnée. Fonctionnant avec des cartes perforées de 90 colonnes et une vitesse de traitement de 6000 , éventuellement 3000 cartes à l'heure, l'ordinateur a réalisé des opérations élémentaires de calcul. II n'y avait pas de fonction de division intégrée. Elle a été mise au point entre 1957 et 1958 à l'Institut des machines mathématiques de l'Académie des sciences de Tchécoslovaquie par une équipe d'Antonín Svoboda. II a reçu le prix d'État pour cette machine.

Cliché Marcela Efmertová

La miniaturisation des ordinateurs a commencé avec l'utilisation des transistors. Les composants ont été intégrés dans des cartes de plus en plus petites (Pinker 2004). La mise au point des microprocesseurs ${ }^{23}$ a fortement accéléré le développement des ordinateurs. Cela s'est traduit en Tchécoslovaquie par la recherche, le développement et la production dans les années 1960, d'ordinateurs analogiques transistors de petite et moyenne taille désignés MEDA T (fig.7). Au milieu des années 1970, le nombre d'ordinateurs vendus de cette série dépassait le millier; ces ordinateurs étaient exportés vers les pays du COMECON. 


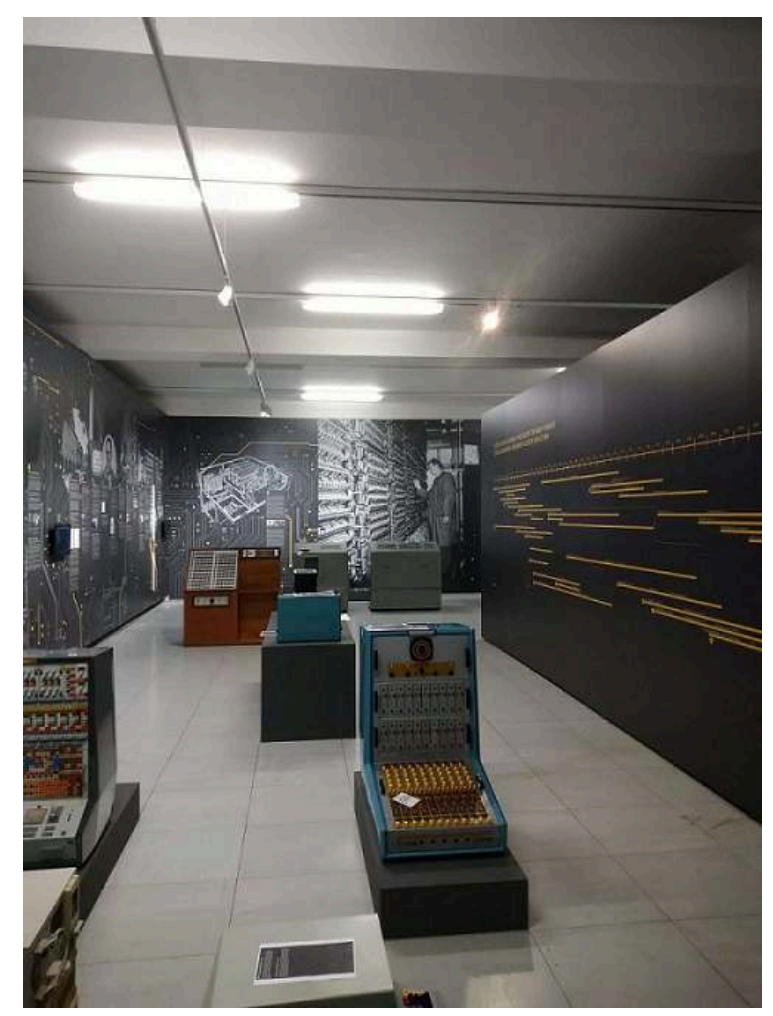

VÚMS et l'entreprise nationale Aritma Praha ont coopéré dans la production de petits ordinateurs analogiques sous vide MEDA 1 et MEDA 2, qui ont commencé à être introduits en série en 1964, par exemple pour la solution d'ensembles d'équations différentielles. MEDA 50 est le plus moderne (et le dernier) de la série d'ordinateurs analogiques MEDA. Destiné principalement aux écoles comme aide à la démonstration, il servait à modéliser des systèmes dynamiques décrits par un ensemble d'équations différentielles, à modéliser des systèmes régulés et des circuits de régulation, à résoudre des ensembles d'équations algébriques, des équations différentielles partielles, etc. Grâce à la combinaison d'un microprocesseur scolaire 8 bits IQ151, un système informatique hybride a pu être conçu. L'ordinateur contenait 6 intégrateurs à trois états, 4 sommateurs, 2 multiplicateurs/diviseurs complets, 4 non-linéarités, 16 potentiomètres manuels, 4 commutateurs analogiques précis, 2 circuits d'échantillonnage et de mémoire, 2 convertisseurs D/A et 1 convertisseur A/D, 3 comparateurs, etc.

Cliché Marcela Efmertová

Puis, les ordinateurs personnels ${ }^{24}$ sont apparus et avec eux les équipements de bureau. Les jeux et les consoles de jeux, les téléphones portables, l'utilisation d'Internet et les fonctionnalités web ont été associés à la problématique dédiée aux technologies informatiques tant ils revêtent une grande importance sociale.

\section{En corollaire : l'organisation d'une collection}

\section{Le cadre légal}

23 La protection du patrimoine culturel matériel dans l'actuelle République tchèque repose sur des concepts énoncés par le ministère de la Culture au profit des institutions muséales (et de leurs archives et bibliothèques) ${ }^{25}$. Grâce à l'adoption de la loi sur la protection des collections des musées ${ }^{26}$ et à un arrêté du ministère de la Culture de la République tchèque $(\mathrm{MC} \mathrm{RT})^{27}$, un cadre législatif a été créé, au profit des collections contemporaines des musées. L'ensemble repose sur le Registre central des collections (RCC), un système d'information unique, accessible au public. Il regroupe une liste de 
collections gérées en vertu de la loi 122/2000 sur la protection des collections des musées. Dans le cadre de l'adhésion de la République tchèque à l'Union européenne, le Département des musées et des galeries (DMG) du ministère de la Culture a été amené à élaborer d'autres lois et notamment celle dénommée 101/2001 sur le retour des biens culturels exportés illégalement, ainsi que la loi 214/2002 relative à l'exportation de certains biens culturels.

Au sein du ministère de la Culture de la République tchèque et sous la responsabilité du DMG, les principales organisations contributives représentent 18 musées, galeries et monuments ${ }^{28}$. C'est pourquoi le DMG a progressivement créé sept lieux de travail ${ }^{29}$ spécialisés au sein des organisations participantes relevant de la compétence du ministère de la Culture. Ils fournissent des informations, proposent des conseils et des services aux propriétaires et administrateurs de collections de musées ainsi qu'à d'autres parties intéressées. De plus, ils publient des instructions sur les méthodes de collecte dans leurs domaines respectifs. Ces documents peuvent aussi être élaborés par les départements universitaires coopérant avec les musées, par exemple sous forme de projets ou de subventions (Efmertová et al., 2019).

Ce cadre comprend également la collecte et la protection d'artefacts dans le domaine des technologies informatiques, de la cybernétique et de l'informatique, pour lesquels les institutions muséales en République tchèque sont plutôt rares. Néanmoins, l'État s'efforce de préserver, de classer et de traiter scientifiquement le matériel informatique en tant que témoignage significatif du développement intellectuel, technique et industriel de la société tchèque des années 1930 à nos jours. Le système qui imprègne désormais l'ensemble de la société à tous les niveaux - technologies de l'information, cybernétique et informatique - est ainsi pris en compte par les musées tchèques.

\section{L'inscription des objets en collection}

La réception d'un objet dans une collection et son inscription au Registre central des collections (RCC) se font sur la base d'une décision de la commission d'experts du ministère de la Culture sur proposition du conservateur. Les documents sont répertoriés sur un recueil dit d'adhésion, en fait un registre d'inventaire (Efmertová et al., 2019). Le cahier d'inscription est relié et les éléments individuels sont numérotés en continu. L'objet doit être enregistré dans les registres d'inventaire dans un délai de trois ans à compter de la date d'inscription de l'objet au dossier chronologique. Au sein de la collection, chaque objet déposé doit avoir son numéro d'enregistrement indiqué sur l'objet afin qu'il ne puisse être éliminé. Le numéro d'enregistrement correspond soit à un numéro d'enregistrement, soit à un numéro d'inventaire.

En règle générale, le dossier doit contenir les informations suivantes :

- le titre et la description succincte de l'objet ;

- la forme d'acquisition de l'objet (don ou achat/vente aux enchères/contrat, avec protocole de remise, collection, legs de l'artefact au musée, échange entre musées) ;

- l'information territoriale et géographique à partir de laquelle l'objet a été obtenu ;

- l'état de l'objet au moment de la remise et l'état actuel ;

- le numéro d'enregistrement (selon le type d'enregistrement - adhésion pour les enregistrements chronologiques ou inventaire pour les enregistrements systématiques) ; 
- la désignation de documents d'archives (conformément à la loi, le document d'archives n'est pas un objet de collection) ou de manuels, de pièces de rechange, etc.

\section{fonction des critères suivants :}

- le lien avec le musée (ou la région) et avec la collection existante ;

- la fonction en termes de besoin social ;

- la coordination des activités de collecte avec d'autres lieux de travail, afin d'éviter les chevauchements ou les doublons ;

- la capacité de traiter l'objet de collection à un niveau professionnel, dans les domaines de collecte et de présentation.

\section{La sauvegarde des objets technologiques après collecte}

\section{Des objets multiples, fragiles et sujets à une dégradation rapide}

Les ordinateurs (personnels, micro-ordinateurs portables ou fixes) ont pris de nombreuses formes, allant des jouets bon marché aux appareils très onéreux. L'ensemble de la technologie informatique se caractérise ainsi en termes de taille par des objets de l'ordre de quelques centimètres à des équipements de plusieurs mètres (par exemple, les modèles des années cinquante). De même, le poids varie du gramme à des centaines de kilogrammes. La grande majorité des objets sont fabriqués avec une large gamme de plastiques (bakélite, pvc, abs, respectivement thermoplastique, thermodurcissable, etc.). Une petite partie est constituée de verre et de divers types d'acier (parfois de fonte), principalement pour soutenir des parties de grandes unités, en particulier dans les unités centrales (Efmertová et al., 2019).

Souvent, lorsque l'artefact est introduit dans la collection, les composants utilisés sont déjà dégradés, en particulier ceux pour lesquels se déroulent des processus physicochimiques continus (condensateurs électrolytiques, batteries et cellules électrolytiques, en contact avec plusieurs métaux). Les pièces mécaniques sont attaquées par la corrosion ou la peinture abrasive; dans le cas des métaux non ferreux, les couches superficielles sont oxydées. Mais la cause la plus fréquente de l'altération vient de l'exposition permanente au soleil et à l'humidité. Les plastiques utilisés dans les équipements informatiques peuvent être dégradés par les rayons UV, les substances organiques déposées à la surface des instruments, etc. D'un point de vue esthétique, les plastiques sont endommagés par une rupture ou des parties manquantes de l'appareil. Parfois, les objets en verre sont rayés, fissurés, cassés ou manquants (Efmertová et al., 2019). Les claviers entrant dans la collection sont généralement très sales; sur les blocs de lettres et de chiffres, de la poussière et des débris se sont accumulés, tels que des cheveux, des restes de boissons et de la nourriture, etc. Certes, un nettoyage de qualité des claviers avec caches hermétiques, etc., n'est pas compliqué à opérer, mais il demande du temps.

31

Les pièces de remplacement peuvent varier en taille et en caractéristiques électriques, de sorte que l'électronique de commande peut ne pas fonctionner correctement, par exemple lorsque l'ordinateur portable a 20 ans ou plus. Leur réparation impose des investissements coûteux pour un musée, d'autant qu'ils n'offrent pas de solution définitive pour une collection pérenne. Le problème le plus courant vient des batteries insérées dans les appareils (Efmertová et al., 2019). Elles ont largement perdu leur capacité à la suite de longues périodes d'utilisation ou de stockage, ce qui grève leur

e-Phaïstos, IX-2 | 2021 
usage dans un musée. Et, dans la plupart des cas, la batterie d'origine, le plus souvent en fin de vie, ne peut pas être remplacée par un modèle actuel. À supposer que l'on trouve une nouvelle source d'alimentation, un problème similaire se produira avec le temps en stockant l'objet dans un dépôt de musée.

La plupart des ordinateurs portables peuvent certes fonctionner sans batterie en les connectant à un réseau. Mais dans certains ordinateurs portables, la batterie est une partie fixe du boîtier extérieur de la machine. Étant donné que la batterie peut couler, il est préférable d'ouvrir le boîtier, de la retirer de la machine et de la stocker séparément. Certains appareils peuvent recevoir un "chien de garde», avec une batterie au lithium intégrée, une horloge en temps réel et une RAM non volatile avec des paramètres de système importants, pour empêcher le démarrage du système en cas de batterie instable ou faible, de manière à protéger l'appareil. Mais ce type d'installation n'est ni simple ni courante et elle est évidemment onéreuse. Des problèmes surviennent également avec le dessous des cartes de circuits imprimés, où des produits chimiques coulent de la batterie et sortent par de petits trous traversant la carte. En conséquence, les deux côtés des plaques sont détruits, et les cartes sont irréparables. En outre, leur environnement est contaminé.

\section{La conception, l'organisation et la technicité des dépôts}

Tous ces problèmes s'accroissent en cas de stockage inapproprié des équipements ${ }^{30}$. Des dégradations apparaissent dans les cas suivants : basse / haute température, humidité / sécheresse, provoquant de la rouille sur les parties métalliques de l'appareil, un environnement sale et poussiéreux, un contact avec des produits chimiques non identifiés, un traitement inapproprié de l'appareil : revêtements de peinture, méthodes de nettoyage inadaptées, etc. (fig.8).

Les phénomènes climatiques peuvent provoquer la formation de taches de moisissures, donnant aux appareils une apparence repoussante, même si elle n'altère pas les fonctionnalités. Ils affectent aussi les parties en caoutchouc de la machine, les fluides d'huile, la mousse en PVC et les rouleaux en caoutchouc des lecteurs de cartes. Les supports magnétiques (par exemple sur les broches du lecteur de cartes pour les calculatrices) utilisaient un cabestan. Après 15 à 20 ans d'utilisation dans la machine, quel que soit le mode et les conditions de stockage, il s'est transformé en une masse collante, spongieuse, qui dégrade l'ordinateur. Les conditions climatiques affectent aussi les câbles de connexion externes ou internes, et possiblement les disques durs. Enfin, la plupart des calculatrices mécaniques motorisées étaient équipées d'un condensateur. Il a tendance à exploser lorsque la calculatrice est allumée après plusieurs années d'inactivité. Ces explosions peuvent être très fortes, produire des vapeurs toxiques et propager des petites particules dans tout le mécanisme 
Fig.8. La difficile sauvegarde des objets technologiques

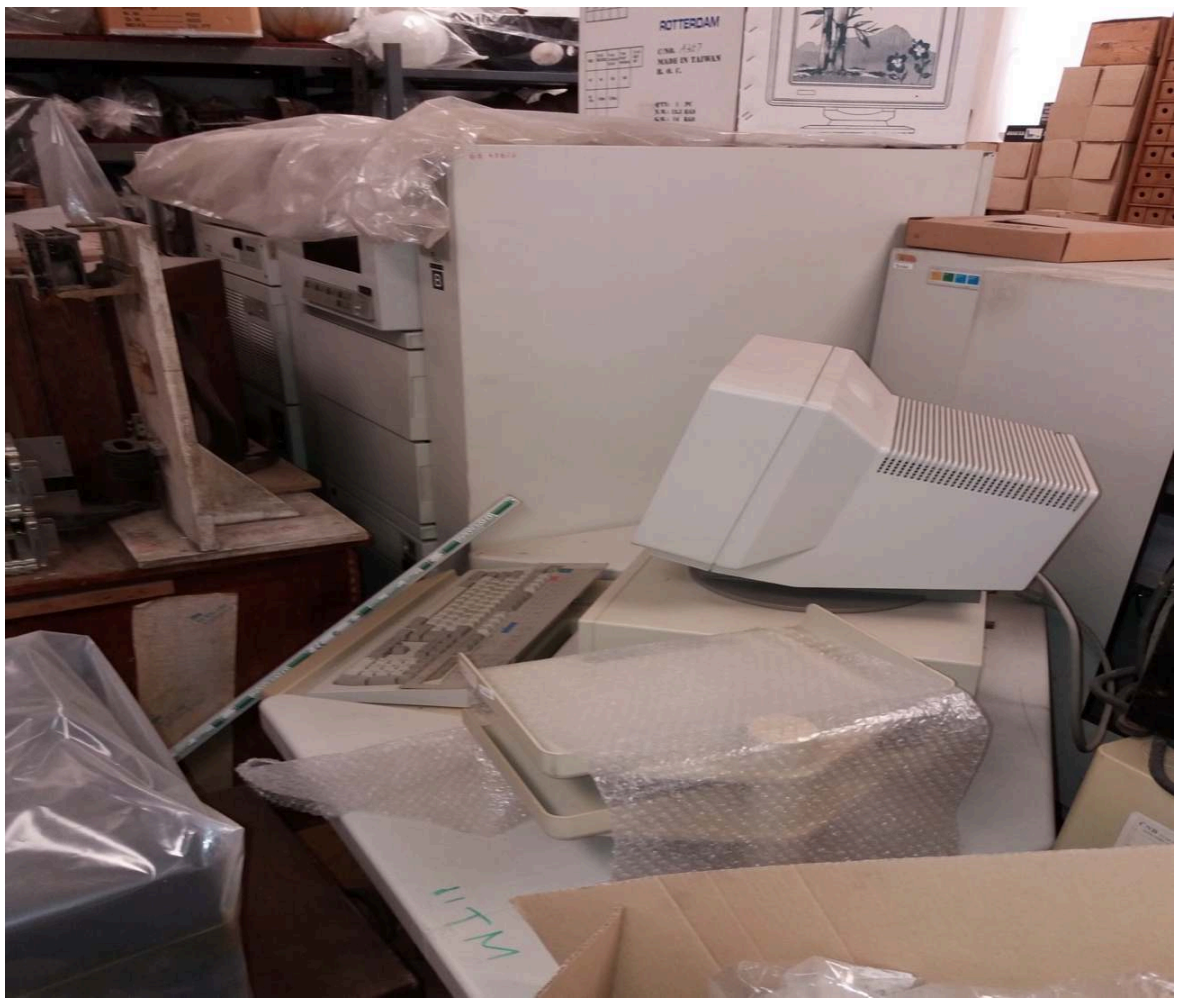




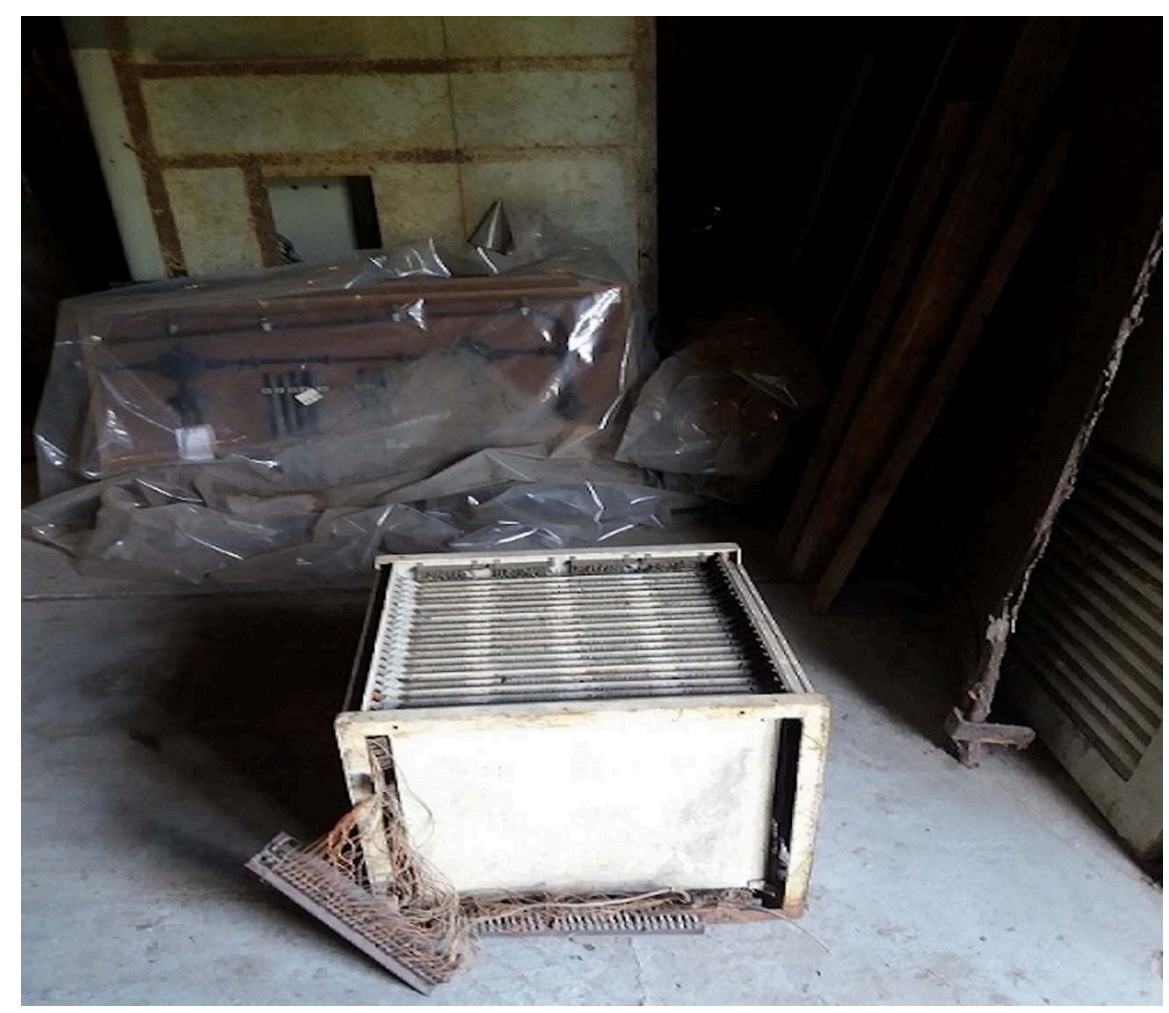

Les clichés documentent le stockage inapproprié d'objets tridimensionnels dans des dépôts. Les conditions d'éclairage ou climatiques : température basse/haute, humidité/sécheresse, sont rarement respectées. Il est très fréquent de trouver dans les dépôts des objets endommagés mécaniquement, moisis, non nettoyés ou non réparés. Le problème le plus courant vient des batteries insérées dans les appareils, des matériaux plastiques, des condensateurs qui ont tendance à exploser ; il faut stabiliser les processus électrochimiques, etc.

Laboratoire d’histoire (électro)technique de la Faculté d'électricité de l'Université polytechnique de Prague

En résumé, les structures internes des circuits électroniques fonctionnent mal en raison d'un ensemble de dégradations liées à leur utilisation et leur usure, sans possibilité de remise en service de l'appareil. Lors de la réception de l'objet dans la collection et de sa mise en dépôt, les processus électrochimiques doivent être stabilisés, de manière à réduire ou arrêter la dégradation elle-même et les appareils doivent subir un traitement de surface adapté.

Les dépôts doivent donc être conçus pour sécuriser autant que possible les objets. Trois sortes de paramètres doivent être pris en compte (Staebler, Wiesmann, 2014) :

- Les paramètres de conception: matériaux de construction; qualité géologique ou géomorphologique de la base du dépôt; infrastructure du bâtiment et sa propre construction; conditions pour les opérations d'utilisation telles que bureaux, salle de recherche, vestiaires et installations sanitaires; équipement d'extinction d'incendie utilisant la norme d'extinction haute performance actuelle HFC227ea - fluorocarbone, etc.,

- Les paramètres d'organisation: chaque dépôt doit être doté de son propre règlement de dépôt, des conditions de son achèvement et de sa distribution ainsi que de son fonctionnement, de son enregistrement, de sa manutention, y compris la manutention manuelle et les machines de manutention de divers types, par exemple les ascenseurs pour le déplacement des œuvres d'art et des collections, des matériaux d'emballage, l'utilisation de cartes électroniques et de systèmes d'enregistrement de caméras d'alarme, de sécurité et de détresse. 
- Les paramètres techniques : il s'agit principalement des systèmes de chauffage par le sol reliés à une chaufferie de type pompe à chaleur, des systèmes de ventilation et de climatisation pour garantir des critères de conservation fixes ${ }^{31}$, en vue de réduire les émissions d'énergie et de carbone associées au fonctionnement des unités de climatisation et en tenant compte des éventuelles pannes, y compris pour ce qui concerne l'utilisation des collections, les systèmes de surveillance et de contrôle, les alarmes anti-incendie, la mesure et la régulation de la température et de l'humidité, éventuellement l'éclairage.

L'objectif est que le dépôt corresponde à un bâtiment pour une collection donnée, sachant qu'il est très difficile d'adapter les bâtiments existants pour accueillir des collections de musées spécifiques (Jirásek 2015).

\section{Pour conclure...}

Fondatrice dans le cadre national tchèque, l'exposition «La piste tchèque dans l'histoire de l'informatique ", et la collection associée ont été conçues dans un cadre multidisciplinaire, tant pour l'électrotechnique que pour l'informatique. L'objectif premier de l'exposition a été de fournir, autour des trois thèmes présentés (Du boulier au smartphone, L'informatique tchécoslovaque, Les micro-ordinateurs et Internet), une sélection de sujets qui évoquent non seulement l'histoire du développement de l'informatique, mais aussi celui des élites techniques, des institutions scientifiques et éducatives, des ministères et entreprises manufacturières impliqués. Les étapes du développement de la technologie informatique en Tchécoslovaquie peuvent être divisées en une période initiale (innovante) 1947-1958, une autre marquée par l'établissement de la recherche et de la production en 1958-1968, suivie d'un programme au sein du Conseil d'assistance économique mutuelle en 1968-1989, et enfin la politique de recherche et développement menée depuis la Révolution de velours. Cette séparation en grandes périodes a servi de base de réflexion pour les institutions de mémoire (nationales) et pour les collectionneurs privés soucieux de créer et de sauvegarder des collections thématiques d'objets informatiques et cybernétiques.

Il s'est donc agi, en tant qu'exposition et collection mère, d'offrir une solution complète à un ensemble de questions liées à de tels projets, en mettant l'accent sur le choix des artefacts. Cela concerne les éléments matériels, périphériques et boîtiers (métaux usagés, plastiques, verre, piles, etc.), dont il faut déterminer les conditions de collecte et d'inventaire, et fournir les recommandations pour leur stockage en dépôts. L'exposition détaille le processus de collecte des objets et explique la manière d'en assurer la description, la préservation pour ensuite parvenir à réunir et valoriser des données historiques en relation avec les technologies cybernétiques, informatiques et de communication.

L'exposition a remporté un grand succès tant auprès des professionnels que du grand public. Elle a également permis un large éventail d'enseignements bien sûr dédiés à l'informatique, la cybernétique et aux TIC, mais aussi à la préservation du patrimoine culturel et matériel tchèque. 


\section{BIBLIOGRAPHIE}

APAY William (ed.), Computing Before Computers, Ames, Iowa State University Press, 1990

ATHERTON A. W., From Compass to Computer : History of Electrical and Electronics Engineering, San Francisco, San Francisco Press Inc., Palgrave MacMillan, 1984 ; DOI : https://doi.org/ 10.1007/978-1-349-17365-5_1

BEAUCLAIR Wilfried de, Rechnen mit Maschinen. Berlin - Heidelberg, Springer-Verlag, 2005 BOYLE Alison, HAGMANN Johannes-Geert (eds.), « Challenging Collections : Approaches to the Herirage of Recent Science and Technology ", Artefacts - Studies in the History of Science and Technology, vol. 11, Washington D. C., Smithonian Institut Scholarly Press, 2017

BROMLEY Allan G., « Charles Babbage's Analytical Engine, 1838 », Annals of the History of Computing, vol. 4, n³, July 1982, p.196 - 217

CERUZZI Paul E., A History of Modern Computing, London - Cambridge, The MIT Press, 2003

ČERNÝ Václav, KLÍR Jiří G., « Antonín Svoboda (1907-1980). Comment une école des sciences a été créée », Vesmír 6, 1991, p.341-345

DENNIS L. Meghan, « An Extension Without an Exhibition Considering the Continued Life (and Usefulness) of a Digital Heritage Output », Digital Reviews, Advances in Archaeological Practice 6(1), 2018, pp. 82-87 ; DOI : https://doi.org/10.1017/aap.2017.35

DOLEJŠÍ Josef, FUČĆK Josef, HAVEL Petr, PERNES Jiří, Sous le drapeau de l'empereur. Prague, Elka Press 2004

DUBBEY John Michael, The Mathematical Work of Charles Babbage, Cambridge, Cambridge University Press, 2004

EFMERTOVA Marcela, Les Personnalités du génie électrique (Antonín Svoboda), Prague, Maison d'édition ČVUT, 1998

EFMERTOVÁ Marcela et al., Méthodologie de l'information et de l'informatique dans le domaine des musées techniques tchèques, Méthodologie certifiée de ČVUT FEL, Prague, MK ČR, 2019, texte disponible sur https ://www.elektropolis.cz/index.php/granty/, consulté le 16 avril 2020

FRK Miroslav, HRBEK Vladimír, L'industrie électrotechnique et électronique tchécoslovaque : 1948-1988, Prague, SNTL, 1988

GILLIES James, CAILLIAU Robert, How the Web was Born : The Story of the World Wide Web, Oxford, University Press, 2000

GOLAN Petr, EFMERTOVÁ Marcela, KONEČNÝ Tomáš, « Czechoslovak Computer School », IEEE Xplore - HISTELCON, 2019, p. 36-39

GOLAN Petr, KOLLINER René, Almanach de l'Institut de recherche sur les machines mathématiques, Prague, 2020

GOLDSTEIN Herman H., The Computer, New Jersey, Princeton University Press, 1972

HADDOCK Thomas F., A collector's guide to Personal Computers and Pocket Calculators 1956-1991, Alabama, Books Americana - Krause Publications, 1993

IFRAH Georges, The Universal History of Computing : From the Abacus to the Quantum Computer, New York, John Wiley \& Sons, 2001 
JANKO Jan, ŠTRBÁŇOVÁ Soňa, La Science de l'époque de Purkyně, Prague, Academia, 1988

JIRÁSEK Pavel, « Les dépôts de musées - conditions de sécurité des dépôts de musées en République tchèque », Bulletin AMG 2/2015 - Dépôts de musées, p. 2-3

KLÍR Jiří G., « L'informatique et les premiers ordinateurs tchèques », dans Qu'est-ce que nos pays ont donné à l'Europe et à l'humanité ?, t.III, Prague, European Literary Club, 2000, p.302-313

KLÍR Jiří George, VYSOKÝ Petr, Ordinateurs de la place Loretánské : vie et œuvre d'Antonín Svoboda, Prague, Maison d'édition ČVUT, 2007

KOTEK Zdeněk, VYSOKÝ Petr, ZDRÁHAL Zdeněk, Cybernétique, Prague, SNTL

KUČERA Jan, KAŠE Jiří, BĚLINA Pavel, Les pays tchèques dans l'histoire européenne, vol. 2, 3, Prague, Paseka, 2006

MATUCHA Jaromír, L'histoire des ordinateurs. Olomouc, École secondaire électrotechnique d 'Olomouc, 2006, p. 4-6

McGRAW-HILL PARKER Sybil P., McGraw-Hill encyclopedia of electronics and computers (McGraw-Hill encyclopedia of science and technology), New York, McGraw-Hill Book Company, 1984

MERGL Ladislav, prof. Dr. Ing. Antonín Svoboda est un scientifique et pédagogue important, fondateur de la technologie informatique tchécoslovaque, Praha, NTM Leaflet, 1997

MINASI Mark, Le Grand guide du hardware, Grada, Prague 2002

NAUMANN Friedrich, Histoire de l'informatique : du boulier à Internet, Prague, Academia, 2009

OBLONSKÝ Jan G., « Eloge : Antonin Svoboda, 1907-1980 », Annals of the History of Computing, vol. 2, No. 4, 1980, p. 284-298, https://doi.org/10.1109/MAHC.1980.10039/

PAVT, Histoire des micro-ordinateurs tchécoslovaques : premiers pas au temps du totalitarisme, Prague, 2014, https ://pctuning.tyden.cz/hardware/multimedia-zvuk-tv/25750-uvaha-copy-pastegoogle-jak-se-ztraci-nase-znalosti ?start =3, consulté le 14 avril 2020

PATTERSON David A. HENNESSY, John L., Computer Organization and Design, San Francisco, Morgan Kaufman, 1998

PETRÁŇ Josef, « La Société royale tchèque des sciences. La place de la société savante tchèque dans l'histoire et le courant de la science ", Vesmír 1995, vol. 74, No. 11, p. 632

PINKER Jiří, Les microprocesseurs et les micro-ordinateurs, Prague, BEN - littérature technique, 2004

PROKEŠ Jaroslav, «Instruction publiée en 1762 pour les tribunaux tchèques et autrichiens ", Bulletin de la Société royale tchèque des sciences, 1926, p. 1-47

RANDELL Brian et al., The Origins of Digital Computers, Berlin-Heidelberg-New York, SpringerVerlag, 1973

RANKIN Joy Lisi, A People's History of Computing in the United States, Hadcover 2018

REILLY Edwin D., Milestones in Computer Science and Information Technology, London, Greenwood Press, 2003

ROJAS Raúl, « How to make Zuse's Z3 a universal computer », Annals of the History of Computing, 1998, vol. 20, p. 5-54

ROJAS Raúl, HASHAGEN Ulf, The First Computers - History and Architectures, London, The MIT Press, 2000 
SANDERS Donald H., Computers in Society, New York, McGraw-Hill, 1977 ; URL : http:// www.worldcat.org/wcidentities/lccn-n79-115408/

STAEBLER Wolfgang, WIESMANN Alexander (eds.), Gut aufgehoben. Museumsdepots planen und betreiben. Landestelle für die nichtstaatlichen museen in Bayern, Berlin - München, Deutscher Kunstverlag, 2014

TANENBAUM Andrew S., Modern Operating Systems, New Jersey, Pearson Prentice Hall, 2009 WILLIAMS Michael R., A History of Computing Technology, Washington, IEEE Computer Society Press, 1997

ZELENÝ Jaroslav, MANNOVÁ Božena, Histoire de l'informatique, Prague, Scientia, 2006

ZUSE, Konrad, Der Computer - mein Lebenswerk, Berlin - Heidelberg, Springer-Verlag, 2007

\section{ANNEXES}

\section{Annexe. Antonín Svoboda (1907-1980)}

Originaire de Prague et pionnier tchèque de la technologie informatique, des ordinateurs et de l'informatique, Antonín Svoboda (14 octobre 1907 Prague - 18 mai 1980 Portland, Oregon, États-Unis) était issu de la famille d'un professeur de l'école industrielle. Après avoir obtenu son diplôme du département de génie mécanique et électrique en 1931, il décide d'étudier la physique théorique et expérimentale à la Faculté des sciences de l'Université Charles de Prague. En 1936, il obtient un doctorat en sciences techniques pour sa thèse de doctorat sur l'utilisation du calcul tensoriel dans la distribution d'énergie. Au moment du service militaire, il était engagé dans le développement et les tests de protection contre les attaques aériennes. Il a commencé à coopérer avec le ministère de la Défense nationale tchécoslovaque sur le développement de viseurs pour la gestion de la défense anti-aérienne. En 1938, avec Jan Vanda, il acheva le concept du viseur, pour lequel ils reçurent un brevet tchécoslovaque. C'est ainsi qu'a commencé la carrière professionnelle et scientifique d'Antonín Svoboda, qui pendant la Seconde Guerre mondiale s'est poursuivie en France sur la défense anti-aérienne et depuis 1943 au Laboratoire de radiation MIT, où il a travaillé avec Norbert Wiener sur de nouveaux systèmes de radar. Au MIT, Antonín Svoboda a élaboré ses méthodes originales de conception de calcul des mécanismes de levier. En 1946, il a utilisé ses connaissances dans le livre Computing Mechanism and Linkages, publié par McGraw-Hill à New York et traduit pour les experts tchèques et russes en 1948 à Prague. En 1948, il a reçu le prix Naval Ordonance Development Award pour le développement d'armes navales. Après son retour en Tchécoslovaquie libérée, il a commencé à construire l'industrie informatique tchèque. Il a recommencé à travailler pédagogiquement et scientifiquement à l'Institut mathématique de l'Université polytechnique de Prague, où il a vite obtenu le titre de professeur associé et a immédiatement introduit au Département de génie électrique théorique et expérimental, dirigé par Zdeněk Trnka, la série de conférences Machines pour le traitement de l'information.

Dans les années 1949 - 1950, Antonín Svoboda a coopéré avec la succursale de Prague de Zbrojovka Brno. Son laboratoire a constitué la base du département de recherche et de 
développement de la société nationale Aritma, dans lequel Svoboda a développé des calculateurs à relais et autres machines mathématiques plus compliquées. Son laboratoire a constitué la base du département de recherche et développement de la société nationale Aritma, dans lequel Svoboda a développé des machines à plaques perforées. En 1953, il a reçu le prix d'État tchécoslovaque pour ses travaux de recherche. Parallèlement, il a travaillé au laboratoire de télémécanique et d'automatisation avec Zdeněk Trnka. En 1950, Antonín Svoboda a accepté un poste à l'Institut central des machines mathématiques de Prague, où il a construit un département de machines mathématiques et, en 1951, il a conçu le premier ordinateur automatique à relais tchécoslovaque, appelé SAPO. Une partie de l'assemblage de cet ordinateur a été mise en service en 1957. Après la création de l'Académie tchécoslovaque des sciences (ATS) en 1953, l'Institut central des machines mathématiques est devenu l'Institut de recherche des machines mathématiques de l'Académie tchécoslovaque des sciences, dont il est devenu directeur. Il a également dirigé le département de recherche et a travaillé au développement d'ordinateurs automatiques EPOS utilisant des éléments logiques électroniques (EPOS 1) et à transistors (EPOS 2, plus tard produits sous le nom de ZPA 600). À cette époque, il a également publié le livre Synthesis of Logic Circuits. Dans la seconde moitié des années cinquante, l'Institut Svoboda se débattait entre les unités académiques et ministérielles, toutes deux contrôlées par le Comité central du Parti communiste tchécoslovaque. En 1958, l'Institut de recherche sur les machines mathématiques a été excommunié de l'Académie des sciences tchécoslovaque, ce qui a rapidement détérioré les conditions du travail scientifique. Ainsi commença une lutte continue de représentants de la science et de la technologie avec le pouvoir communiste. Le travail de Svoboda sur les machines mathématiques ne pouvant pas continuer selon ses idées, il décida de quitter la Tchécoslovaquie pour la deuxième fois en 1964. En 1965, il s'installe définitivement aux États-Unis. Il commença à travailler au General Electric Research Center à Phoenix, Arizona. Un an plus tard, il rejoint l'Université de Californie de Los Angeles, où il est nommé professeur en 1968. Dans les années soixante-dix, il s'est entièrement concentré sur le travail universitaire. En 1975, en tant que citoyen américain, au cours de son cycle de conférences à Paris, qui a culminé sa longue carrière scientifique et professionnelle, il a brièvement et pour la dernière fois visité sa Tchécoslovaquie natale.

\section{NOTES}

1. Le comte F. J. Kinský, avec l'aide de l'homme des lumières, minéralogiste et malacologiste Ignác Born (1742-1791) a fondé la collection minéralogique qui est devenue la base du cabinet d'histoire naturelle de l'université de Prague.

2. Les membres fondateurs étaient des personnalités importantes de la science des pays tchèques - Ignác Born (minéralogiste, géologue, malacologiste, franc-maçon), Josef Sonnenfels (avocat, théoricien de l'absolutisme des Lumières), Gelasius Dobner (piariste, fondateur de l'historiographie tchèque moderne), Karel Rafael Ungar (bibliothécaire, prêtre), Mikuláš Voigt (linguiste, historien littéraire, numismate), Karel Jindřich Šteib (éducateur, historien), František Josef Kinský (politique, avocat, scientifique et pédagogue militaire) ou Ignác Cornova (jésuite, poète).

3. Le Royaume tchèque ou le Royaume de Bohême (en latin Regnum Bohemiae) était un État dirigé par un roi en Bohême du XIII ${ }^{\mathrm{e}}$ siècle à 1918. D'autres pays historiques ont été associés au 
Royaume de Bohême à diverses époques, en particulier le Margraviat de Moravie, la Principauté de Silésie et la Lusace. Les pays sous la domination du roi tchèque sont collectivement appelés les pays de la Couronne tchèque. Le titre royal avait déjà été accordé à certains princes de la dynastie des Přemyslides, mais la transformation de la Principauté tchèque en royaume héréditaire a finalement été confirmée par la Bulle d'or de Sicile en 1212. Le trône royal tchèque était gouverné par la dynastie des Přemyslides (1198-1306), les Lucembourg (1310-1437), la Maison Jagellon (1471-1526), Habsbourg (1526-1780) et Habsbourg-Lorraine (1780-1918). Depuis 1526, le Royaume tchèque était une partie autonome de la monarchie des Habsbourg qui en 1867 a été divisée en Cisleithanie et Transleithanie, soit une organisation double de la monarchie austro-hongroise. Cette organisation a duré jusqu'à la fin de la Première Guerre mondiale, lorsque le 28 octobre 1918, le royaume et la monarchie ont cessé d'exister et la République tchécoslovaque indépendante a été proclamée.

4. Le centre intellectuel de la Silésie autrichienne a été renforcé à Opava et le plus ancien musée public sur le territoire de l'actuelle République tchèque a été créé. Le public a pu rencontrer un nouveau phénomène culturel et éducatif dès le 1er mai 1814. Trois personnalités - le professeur du lycée d'État d'Opava Faustin Ens, le gouverneur à la retraite Franz Mückusch von Buchberg et le burgrave d'Opava Johann Johann Schößler - ont contribué à la réalisation du projet. À cette époque, la collection du musée contenait principalement du matériel scientifique qui, cependant, était complété par des preuves matérielles de l'histoire et de la préhistoire, et des modèles de diverses installations de production et de mécanismes mécaniques. La collection du musée s'est élargie non seulement par des collections sur le terrain, mais aussi par des dons et des achats, provenant également d'autres pays. La riche bibliothèque faisait partie intégrante du musée depuis le début. Les citoyens d'Opava et la noblesse silésienne considéraient le musée comme un moyen efficace d'améliorer le prestige de la ville et du pays lui-même. Voir http://www.szm.cz/ rubrika/5/muzeum/historie.html, consulté le 3 mars 2020.

5. En 1814, le plus haut burgrave tchèque d'alors, František Antonín, comte Libštejnský de Kolovraty (1778-1861), a demandé à l'un des chercheurs vivants les plus importants de l'époque, le botaniste et paléontologue Kašpar Maria comte de Šternberk (1761-1838), la création et la gestion du Musée patriotique tchèque. L'idée a été réalisée à partir des collections de sciences naturelles de Šternberk (au nombre de 145000 articles) en 1818, lorsque la fondation de l'actuel Musée national a été engagée le 15 avril. La partie sociale et scientifique du musée était dirigée par un jeune historien tchèque et plus tard homme politique tchèque important: František Palacký (1798-1876). La Société du Musée patriotique de Bohême, dirigée par Šternberk et Palacký, a été créée à l'époque pour la conception et le développement du musée. Elle fonctionnait en tant que propriétaire et gestionnaire des collections et comme organisatrice de tous les travaux du musée. La fondation de la première Revue scientifique tchèque du Musée national (1827) et la fondation de la Matrice tchèque (1831), qui ont contribué à la publication d'ouvrages de base de la science et de la littérature tchèques, ont été d'une grande importance pour le musée et toute la vie scientifique en Bohême. Voir https://www.nm.cz/o-nas/historie, consulté le 3 mars 2020.

6. Le développement des sciences naturelles cultivées dans les institutions scientifiques, éducatives, religieuses et muséales devait contribuer à la croissance économique des pays tchèques. La connaissance du développement historique devait alors favoriser une meilleure compréhension de l'époque actuelle et, aux yeux de la noblesse, promouvoir le patriotisme foncier comme contrepoids au centralisme bureaucratique et à l'absolutisme de l'État. Le Musée de pays de Brno, nommé en l'honneur de l'empereur François $\mathrm{I}^{\mathrm{er}}$, avait également ces objectifs. La naissance du musée est due aussi à Christian Carl André (1763-1831), homme des lumières de Moravie et économiste national - promoteur de l'économie, au journaliste, chancelier de la société moravo-silésienne pour l'amélioration du labourage, des sciences naturelles et de la civilisation, Josef Earl Auersperg (1767-1829), avocat, diplômé de l'Université Charles de Prague, 
homme des lumières, membre de la Loge des Franc-Maçons, partisan de la Révolution française, éminent représentant des intellectuels moraves, partisan de la société économique, gouverneur, puis Antonín Bedřich Mitrovský (1770-1842), avocat, haut fonctionnaire qui, en tant que gouverneur de province, a déployé des efforts pour la création d'un musée régional, grâce auquel le musée a acquis la Cour épiscopale de Brno comme premier bâtiment et au comte Hugo František Salm-Reifferscheidt (1776-1836), érudit éclairé, entrepreneur minier, en métallurgie et agriculture, directeur de la société moravo-silésienne pour l'amélioration du labourage, des sciences naturelles et de la civilisation. Il a fait don au musée des collections de produits naturels et de nombreux objets d'art. Tiré de http://www.mzm.cz/historie-museum, consulté le 3 mars 2020.

7. Visite virtuelle et autres informations Voir https://www.nm.cz/o-nas/historie, consulté le 3 mars 2020.

8. Il s'agit de la plus grande institution tchèque spécialisée dans les collections techniques de musées. Le musée a été fondé le 5 juillet 1908 en tant que musée technique du Royaume de Bohême. À l'heure actuelle, il présente ses collections dans 21 expositions et dans un certain nombre d'expositions temporaires, dans un bâtiment à Letna, Prague, au Centre du patrimoine architectural de Plasy et au dépôt ferroviaire de Chomutov (tous deux en Bohême occidentale). Les débuts de la collecte des objets techniques sur le territoire tchèque sont liés à l'Union pour l'encouragement de l'industrie en Bohême, qui a créé en 1834 un cabinet technique dans lequel elle a recueilli des échantillons de machines et de produits industriels. Vojtěch Náprstek, fondateur des musées techniques modernes tchèques, a établi une collection d'innovations industrielles et techniques mondiales à partir de 1862. En 1887, il a ouvert les collections du Musée industriel tchèque dans une nouvelle partie de la maison U Halánků au centre de Prague. Le succès des expositions au XIX ${ }^{\mathrm{e}}$ siècle et au début $\mathrm{du} \mathrm{XX}^{\mathrm{e}}$ siècle, les efforts pour préserver les preuves du développement de l'industrie et de l'artisanat tchèques et ceux pour soutenir un tel musée de la Capitale de la monarchie de Vienne ont permis la création du Musée technique du Royaume tchèque à Prague. Les activités de collecte de l'école d'ingénieurs des États de Prague (depuis 1707) et les activités connexes de l'Institut polytechnique Gerstner (depuis 1806) ont ensuite été incluses dans le musée. Le musée a été fondé le 5 juillet 1908. En 1910, une association muséale a été créée et, le 28 septembre 1910, le musée a inauguré sa première exposition au Palais Schwarzenberg de Prague, sur la place Hradčanské. À partir de http://www.ntm.cz/ muzeum/historie-museum, consulté le 3 mars 2020).

9. Voir https://www.psp.cz/docs/laws/listina.html, consulté le 5 avril 2020.

10. Il s'agit principalement de collections privées - par ex. de M. Petr Váradi de Stará Paka ou de Michal Suchánek de Vysoké Mýto (Voir http://kormus.cz/mvt/pocitace/michal_suchanek.php ou http://cs-pocitace.ic.cz/, consulté le 23 mars 2019), ou expositions au Musée technique de Brno (http://www.technicalmuseum.cz/expozice/vypocetni-technika/, consulté le 23 mars 2019), au Centre de calcul de l'Académie slovaque des sciences de Bratislava (http:// www.vystava.sav.sk/, consulté le 23 mars 2019) ou des expositions occasionnelles dans des écoles de tous types (par ex. au collège professionnel et au lycée industriel de Ž̌̌ár nad Sázavou, http:// cs-pocitace.ic.cz/, consulté le 23 mars 2019), à la faculté des technologies d'information de l'Université polytechnique de Brno, https://www.fit.vut.cz/units/museum/.cs, consulté le 23 mars 2019), qui ne se consacrent pas systématiquement à cette activité. Un autre exemple se manifeste à travers l'exposition dans le cadre du Laboratoire d'histoire (électro)technique de la Faculté d'électricité de l'Université polytechnique de Prague, où deux expositions d'informatique ont été installées en 2016 et 2018. Leur succès a permis l'obtention de la subvention du ministère de la Culture de la République tchèque NAKI II, intitulée « Le siècle de l'information : le monde de l'informatique et du génie électrique - le monde informatique en nous » (DG18P02OVV052 pour la période 2018-2021), consacrée non seulement à la collecte, à la recherche historique et à la présentation de tels artefacts, mais aussi aux activités de collecte des technologies informatiques, 
cybernétique et informatique (voir note 6). Voir https://www.elektropolis.cz/index.php/ fotogalerie/nggallery/historicka-laborator-elektrotechniky/vystava-stoleti-informace-hle-felcvut-v-praze-2016 ou https://ekonom.feld.cvut.cz/cs/katedra/laboratore/historicka-laboratorelektrotechniky/ ou http://www.odbornecasopisy.cz/elektro/clanek/historii-vypocetnitechniky-predstavi-unikatni-vystava-na-fakulte-elektrotechnicke-cvut-v-praze--1634 ou http:// www.odbornecasopisy.cz/clanek/fakulta-elektrotechnicka-cvut-v-praze-opet-predstavi-historiivypocetni-techniky--1915 ou https://www.fel.cvut.cz/cz/vz/tz/2016-0516-TZ-FELCVUTvystava_historickych_pocitacu_final.pdf ou https://www.blesk.cz/clanek/regiony-praha-prahavolny-cas/395309/na-cvut-si-muzete-prohlednout-historicke-pocitace-i-unikaty-ze-70-let.html ou https://www.parlamentnilisty.cz/zpravy/tiskovezpravy/Fakulta-elektrotechnicka-CVUT-vPraze-predstavi-zajemcum-o-studium-moderni-techniku-i-jeji-historii-471270, consultés le 23 mars 2019.

11. Voir https://www.computerhistory.org, consulté le 8 mai 2020.

12. Voir https://www.computerhistory.org/exhibits/revolution/, consulté le 8 mai 2020.

13. Voir sciencemuseum.org.uk, consulté le 8 avril 2020.

14. Voir https://www.hnf.de/dauerausstellung/ausstellungsbereiche/nixdorf-wegbereiter-derdezentralen-datenverarbeitung.html, consulté le 18 février 2021.

15. Voir https://www.bbox-actus.com/news/565, consulté le 20 mai 2019.

16. Philippe Nieuwbourg est spécialisé en science des données depuis 1995 (Business Intelligence, Data Warehouse, Big Data, Intelligence Artificielle, Machine Learning...). Il est analyste, éditeur, chercheur et consultant-formateur. Selon lui, les données constituent le pétrole du XXI ${ }^{e}$ siècle. Chaque entreprise doit savoir comment collecter, stocker, analyser et présenter des données. Il est le créateur et directeur du site français de référence Decideo (www.decideo.com, www.decideo.fr) sur l'intelligence économique (depuis 1995) et du projet de recherche international Algorithmic Economy (depuis 2018), qui évalue la valorisation des actifs incorporels, des données et des algorithmes de la société. Il est également membre du réseau d'analystes indépendants BBBT (Boulder Business Intelligence Brain Trust), créé par Claudia Imhoff. Voir https://www.nieuwbourg.com, consulté le 21 mai 2019.

17. http://www.aconit.org.

18. Voir webarchiv - https://www.webarchiv.cz/cs/dokumenty, consulté le 8 mai 2020.

19. Articles sur les expositions de M. Petr Váradi - voir https://www.denik.cz/kralovehradecky$\mathrm{kraj} / \mathrm{sberatel-petr-varadi-ma-doma-kremikove-nebe-20121122-hxlm.html,} \mathrm{http://}$ www.muzeumusti.cz/c1537/POCITACOVY-DAVNOVEK/, https://www.youtube.com/playlist? list=UUHdaK4VTFz9f2iG4hAKNg_Q, $\quad$ https://sever.rozhlas.cz/v-usteckem-muzeu-si-naexponatech-muzete-zahrat-pocitacove-hry-popularni-v-80-6864929, http://pakvim.net/user/ UCHdaK4VTFz9f2iG4hAKNg_Q, consultés le 8 mai 2020.

20. Sur la collection de M. Michal Suchánek voir http://cs-pocitace.ic.cz/, p. 32 , consulté le 8 mai 2020.

21. Promenade virtuelle à travers l'exposition en tchèque : voir https://www.youtube.com/ watch?v=wNXAsFnUkuI, consulté le 18 février 2021.

22. Elle se concentre sur plusieurs domaines : A. chronologie, B. manuels et documentation, C. premiers instruments - boulier, etc., supports d'enregistrement et lecteurs, logiciels, processeurs et mémoires et autres matériels, ordinateurs et terminaux vidéo, composants externes moniteurs, claviers, imprimantes, scanners, D. mise en réseau, E. divers - tubes, modules, vidéos pédagogiques, par exemple sur la programmation, les vidéos, etc.

23. Le Microprocesseur (unité centrale de traitement) est un équipement programmable polyvalent qui accepte les données numériques en entrée, les traite avec des instructions stockées et affiche le résultat en sortie. Un microprocesseur est un exemple de circuit logique séquentiel qui utilise un système binaire pour stocker des données. 
24. Un ordinateur personnel (Personal Computer, PC) est un ordinateur dont le prix d'achat, la taille et la capacité de travail sont adaptés pour être utilisé par un individu. À l'origine, ce terme désignait un ordinateur de bureau, c'est-à-dire un boîtier auquel sont le plus souvent connectés un écran, un clavier et une souris. D'autres périphériques peuvent être également associés : des haut-parleurs, une webcam ou un joystick. L'ordinateur personnel peut être également de type portable - laptop, notebook ou netbook. BESTOFMEDIATEAM, Computer History 101: The Development of The PC, 2011. Voir http://www.tomshardware.com/reviews/upgrade-repair-pc, 3000-3.html, consulté le 8 mai 2020.

25. Conception du développement des musées en République tchèque 2015-2020. Ministère de la Culture 2015. Le 20 août 2015, le Gouvernement de la République tchèque a approuvé, dans sa résolution $n^{\circ} 655$, le concept de développement des musées en République tchèque de 2015 à 2020 . C'est le premier document complet pour le développement des musées contemporains en République tchèque. Il se concentre sur le soutien systématique, efficace et efficient du patrimoine national naturel, culturel et technique à travers les collections des musées. Le document s'appuie également sur les concepts antérieurs d'une prise en charge plus efficace du patrimoine culturel mobilier de la République tchèque pour les années 2003 - 2008 et 2010-2014. Voir https:// www.mkcr.cz/koncepce-rozvoje-muzejnictvi-v-ceske-republice-1594.html, consulté le 3 mars 2020.

26. Loi 122/2000. Voir https://www.zakonyprolidi.cz/cs/2000-122, consulté le 3 mars 2020.

27. Arrêté 275/2000. Voir https://www.zakonyprolidi.cz/cs/2000-275, consulté le 3 mars 2020.

28. Voir https://www.mkcr.cz/strucna-historie-oddeleni-a-jeho-aktivit-373.html, consulté le 3 mars 2020.

29. Ibidem.

30. Une question importante pour l'activité de collecte est l'état des artefacts. Le problème est traité plus en détail avec la documentation iconographique qui a été également utilisée dans cet article, https://ub.fnwi.uva.nl/computermuseum//rampspoed.html consulté le 8 mai 2020.

31. ČSN EN 15757 : Protection du patrimoine culturel - Exigences de température et d'humidité relative de l'environnement pour prévenir les dommages mécaniques sur les matériaux hygroscopiques organiques résultant du climat, adoptées en République tchèque en 2011. Une autre norme généralement acceptée pour la classification du microclimat muséal est la recommandation mentionnée au recueil de la Société américaine des ingénieurs pour le chauffage, la réfrigération et la climatisation (ASHRAE Handbook 2007), traitée en coopération étroite avec l'Institut canadien de conservation. La classification de l'environnement dans les musées, les galeries, les archives et les bibliothèques est divisée dans le cadre des notations AA - D, qui définissent les fluctuations saisonnières et à court terme acceptables de RV et $T$, en relation directe avec les risques et avantages associés pour la conservation des objets. Voir aussi l'article « Les normes actuelles du microclimat dans la pratique muséale ", Forum des conservateursrestaurateurs, Prague 2014. Des informations complémentaires à ce sujet sont fournies par le Centre méthodique de conservation du Musée technique de Brno - Voir http:// www.mck.technicalmuseum.cz, consulté le 14 avril 2020.

\section{RÉSUMÉS}

L'article se concentre sur la présentation et la préparation de l'exposition « La piste tchèque dans l'histoire de l'informatique ", proposée au musée national de Prague en 2020. Il s'agit de la 
première exposition de ce type dans les musées techniques tchèques. Le Laboratoire d'histoire (électro)technique de la Faculté d'électricité de l'Université polytechnique de Prague, initiateur du projet, a dû développer une méthodologie pour la collecte, la protection, les caractéristiques et la recherche scientifique, puis pour ajuster les objets tridimensionnels - machines spécialisées pour l'informatique, la communication, la cybernétique et les technologies de l'information. Le but de l'exposition, alors que tout le monde côtoie au quotidien un smartphone ou un ordinateur personnel pour le travail ou le divertissement, est de présenter non seulement ces appareils, mais aussi l'élite des ingénieurs, tchèques notamment, à l'origine de cet épanouissement, en faisant remonter les racines au XVIIIe siècle.

This article discusses preparations for and installation of an exhibition on computer technology, cybernetics and information science in the Czech Republic (Czech lands). It is essentially a first exhibition of this type in Czech technical museums, which was launched by the Laboratory of (electro) technical history of the Faculty of Electricity at the Polytechnic University of Prague and which had to develop a methodology and a certified method for the collecting, protecting, scientifically classifying and examining and, last but not least, adjusting for display 3D exhibits specialized machines for computer, communications, cybernetic and information technology. Seen from today's perspective, when virtually everybody is capable of handling a smartphone or using a computer for work and entertainment, the purpose of the exhibition is not only to trace the development of those machines but also to introduce the engineering elite standing at the cradle of this technology since the 18th century.

\section{INDEX}

Mots-clés : histoire des techniques, histoire des sciences, informatique, ordinateur, cybernétique, musée

Keywords : history of technology, history of science, computer, cybernetics, museum

\section{AUTEUR}

\section{MARCELA EFMERTOVÁ}

Historienne en sciences et des techniques, directrice d'études à l'Université polytechnique de Prague, Marcela Efmertová y dirige le laboratoire d'Histoire (électro)technique de la Faculté d 'électricité. Spécialisée en histoire tchèque (histoire des techniques et de l'électrotechnique) dans le contexte européen des $19^{\mathrm{e}}$ et $20^{\mathrm{e}}$ siècles, elle est l'un des auteurs de l'exposition « La piste tchèque dans l'histoire de l'informatique ». 